

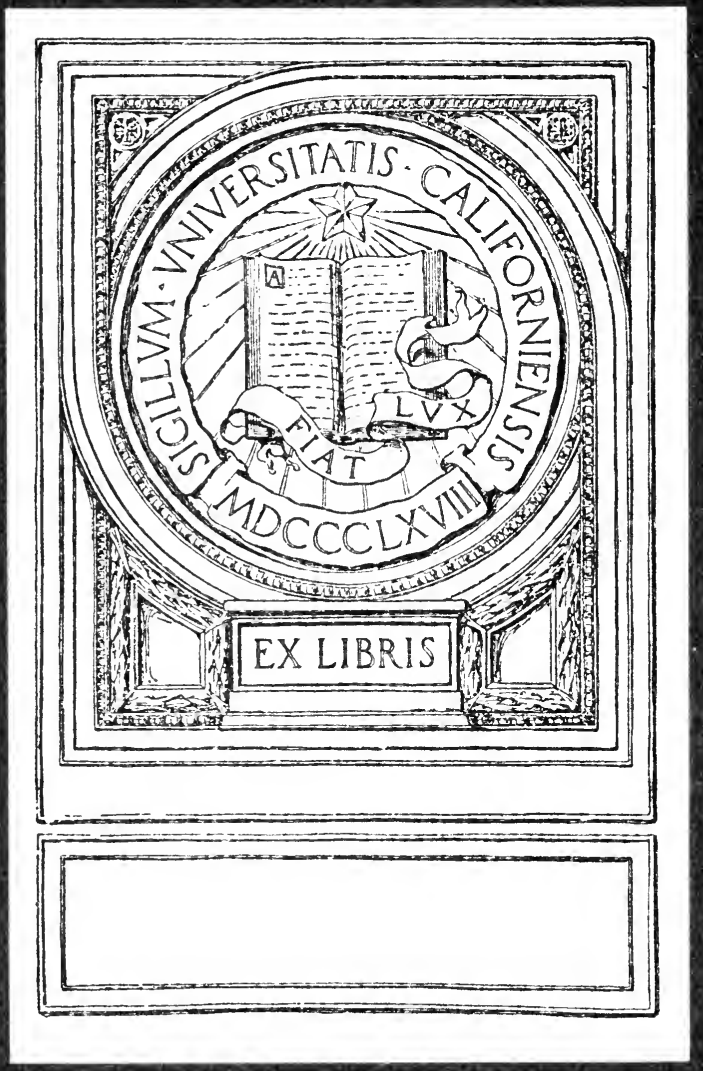




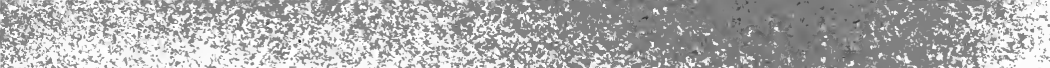

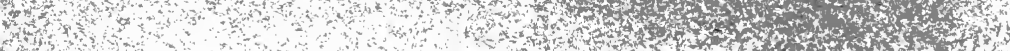

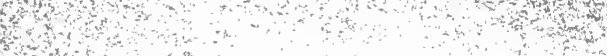

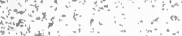

int 


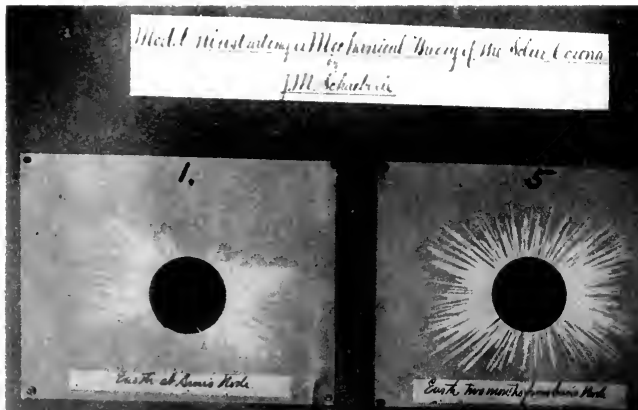

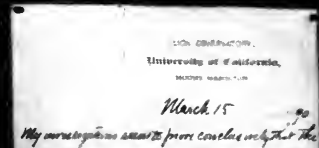

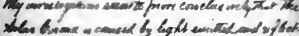

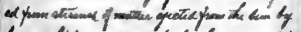

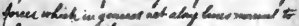

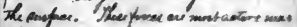
The mene of enol nanstyone.

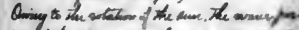

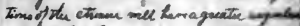

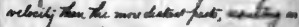

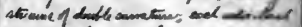

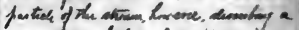

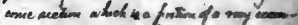

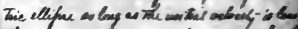

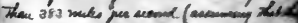

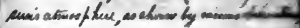

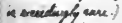

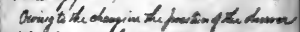

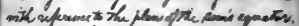

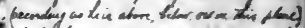

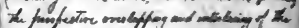

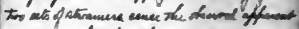

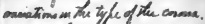

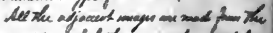

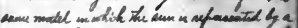

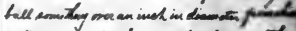

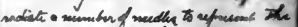

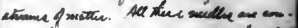

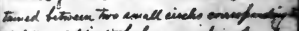

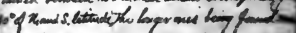
the wisitle of each gone and eligh

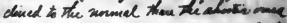
in onder that the mone decturf porene of the

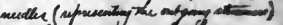

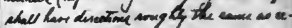
quined ty flyoine ber.

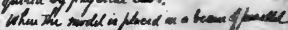

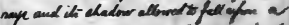

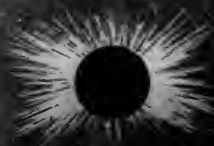

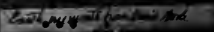

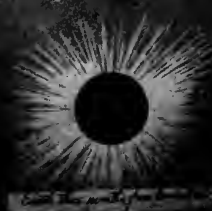

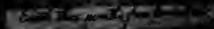

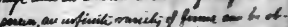

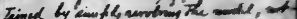

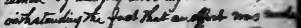

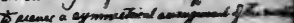

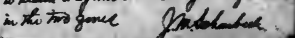




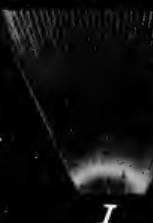

$I$

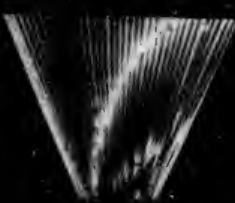

II
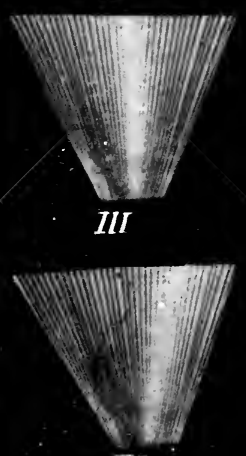

$$
\text { IV }
$$

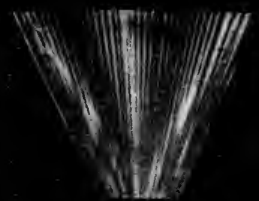

$\boldsymbol{V}$.

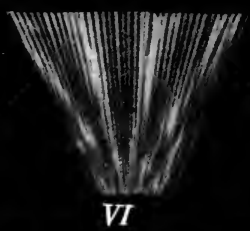

VI
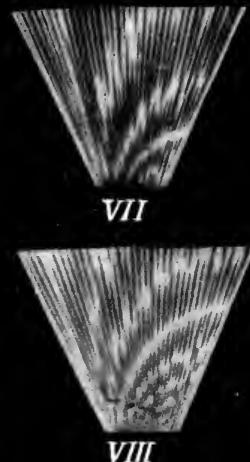

VIII

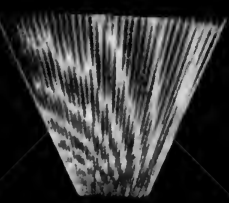

IX

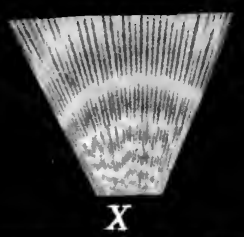

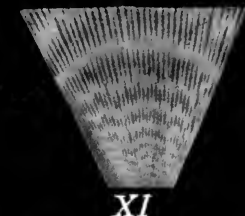
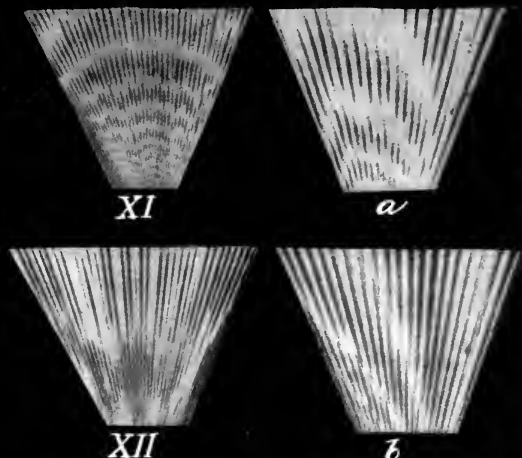

子
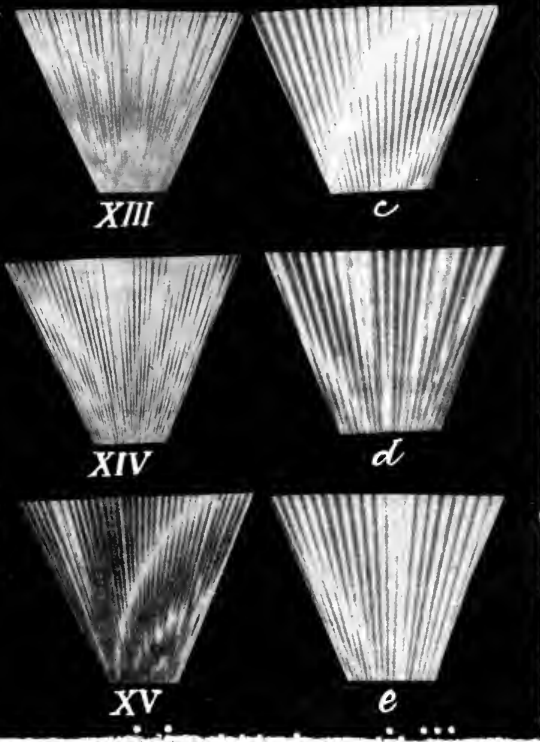

Models illustrating the method of formation of the Polar Rays. 
$\because 4 \quad \because$

- 


\section{A MECHANICAL THEORY}

OF THE

SOLAR

CORONA.

By J. M. SCHAEBERLE.

SACRAMENTO:

STATE OFFICE, : : A. J. JOHNSTON, SUPT. STATE PRINTING. I 89 I . 
add'l.

QE529 


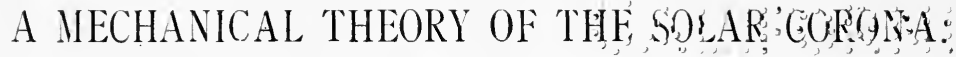

By J. M. Schaeberle.

The essential principles which lie at the foundation of the theory here advanced were first published last spring in a short note entitled "A Mechanical Theory of the Solar Corona." (Publications, A. S. P., No. 7; Mon. Not., R. A. S., Vol. 4, p. 372.) One year previous to this time (in February, 1889), I expressed my belief to Professor HoLDEN and other astronomers at this Observatory, that the coronal form was produced by streams of matter ejected from the lower latitudes of the Sun. At the time Professor Holden desired me to work up the subject, and offered to give me any assistance in his power; but as I had not given much attention to solar observation, and was still less familiar with the physical researches of those who made the Sun a special study, I did not deem the subject a proper one for me to cope with at that time, although I intended to attack the problem later on.

Through the liberality of Colonel Crocker, who offered to bear the expense of an expedition to South America, to observe the solar eclipse of December 21-22, this problem of the solar corona was to rivet my attention sooner than I had anticipated.

The highly satisfactory results secured by the Lick Observatory party, consisting of S. W. Burnham and J. M. Schaeberle, at Cayenne, South America, were such that after the development of our plates the views which I had held for nearly a year were very much strengthened.

By the time we again reached the Lick Observatory the superstructure of the present theory was built, and it only required the finishing touches relating to the more minute details of the corona, and a comparison with published observations, to bring the matter to its present shape.

In what follows I shall present the theoretical investigations in as simple and brief a manner as possible. 
Three well known facts serve as a basis on which the whole theory rests. They are:

1. The eruptions of the Sun's surface are most active and

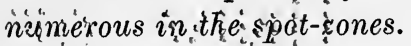

2."The Sun rotates about an axis passing through its center.

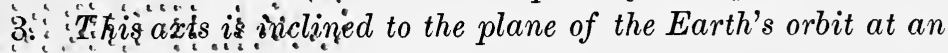
angle of about $82 \frac{3}{4}^{\circ}$.

As the present theory also enables one to reproduce, graphically, practically all the phenomena observed during a total eclipse of the Sun, and as the graphical construction is in strict accordance with known mechanical laws, the following brief statement of the theory may be properly made here:

The theoretical corona is caused by light emitted and reflected from streams of matter ejected from the Sun, by forces which, in general, act along lines normal to the surface of the Sun; these forces are most active near the center of each Sun-spot zone.

Owing to the rotation of the Sun, the streams of matter will not lie along normals, since the angular velocity of different portions of a stream grows less as the distance from the Sun increases; in other words, the streams are of double curvature. Each individual particle of the stream, however, describes a portion of a conic section, which is a very elongated ellipse, so long as the initial velocity is less than three hundred and eighty-three miles per second (assuming that the Sun's atmosphere, as shown by various observations, is exceedingly rare).

Certain variations in the type of the corona admit of an exceedingly simple explanation, being principally due to the change in the position of the observer with reference to the plane of the Sun's equator. According as the observer is above, below, or in the plane of the Sun's equator, the perspective overlapping and interlacing of the streamers cause the observed apparent variations in the type of the corona.

The general direction in which these ejective forces act will be along radii. There may, of course, be numerous particular exceptions to this general direction.

Similarly, since observation shows that certain solar motions are apparently confined to special regions, which are symmetrically situated with reference to the axis of rotation of the Sun, we are evidently justified in assuming that the forces which cause these motions are, in general, uniformly distributed 
around each circle of latitude, since there is no a priori reason why, in a highly heated rotating mass of matter, the solar activity should be confined to any particular circles of longitude. There may, of course, be particular exceptions to a uniform distribution of the forces.

As the exact nature of the atmosphere surrounding the Sun is unknown, and as its extreme rarity has been demonstrated by various observations, ${ }^{*}$ the heliocentric motions of all particles exterior to the Sun's visible surface will be considered to be unimpeded by solar atmospheric resistances.

What precedes may be taken to be a complete statement of the general features of the mechanical theory of the corona. In order to compare it with observation it will be necessary to

*Notes. -With reference to the views of solar physicists on the subject of the Sun's atmosphere, I quote a few paragraphs giving the most recent conclusions:

Professor Young, in his General Astronomy, says: "The corona cannot be a true 'solar atmosphere' in any strict sense of the word. No gaseous envelope in any way analogous to the earth's atmosphere could possibly exist there in gravitational equilibrium under the solar conditions of pressure and temperature. The corona is probably a phenomenon due somehow to the intense activity of the forces there at work; meteoric matter, cometic matter, matter ejected from the Sun, are all concerned.

"That this matter is inconceivably rare is evident from the fact that in several cases comets have passed directly through the corona without experiencing the least perceptible disturbance of their motions. It is altogether probable that at a very few thousand miles above the Sun's surface, its density becomes far less than that of the best vacuum we can make in an electric lamp."

The following paragraphs are from Mr. KEELER's Report on the Solar Eclipse of January 1, 1889 (published by the Lick Observatory):

"Spectroscopic observation furnishes us with many facts which cannot be reconciled with the theory of an extensive solar atmosphere. Two of the most important objections drawn from this source are given below; the first has long been recognized as especially perplexing:

"(1) The pressure at the surface of the chromosphere is shown by the spectroscope not to exceed that of a few millimetres of mercury, and this we must accept as the pressure due to an atmosphere from half a million to a million of miles deep, notwithstanding the fact that the force of gravity is twentyseven times as great at the surface of the Sun as at the surface of the Earth.

"(2) According to theory, as well as observation, the upper limits of the gaseous envelopes of the Sun ought to be ordered according to their densities. The material which produces the $1474 \mathrm{~K}$ line, and which may always be seen in the chromosphere spectrum, is, according to this criterion, as unmistakably denser than hydrogen as is magnesium vapor or iron vapor; but if we accept the coronal spectrum as evidence of the existence of an atmosphere, we are, by exactly the same principle, driven to the same conclusion that the $1474 \mathrm{~K}$ material is far less dense than hydrogen. The contradiction could not be more abrupt and inexplicable."-Hastivgs. 
deduce the laws which govern the appearances of a typical corona produced by solar eruptions of the character already described. For convenience, I have made no distinction between the real and these theoretical solar phenomena; it should therefore be borne in mind that in the discussion for deducing the laws of coronal phenomena this theoretical corona is always the one referred to.

We shall first inquire into the form of the trajectory of a particle ejected from the Sun by a force sufficient to remove it one or more diameters from the Sun's surface.

If there were no motion of rotation, all particles ejected from a given point on the Sun's surface would, in general, lie along a normal passing through this point. The rotation of the Sun, however, introduces other forces, the resultant of which can, without appreciable error, be regarded as acting at right angles to both the axis of rotation and the line along which the ejective force acts.

In considering the motion of an ejected particle:

Let $T_{\circ}$ denote the time of one rotation of the Sun (in latitude $\phi$ ).

Let $R_{\mathrm{o}}$ denote the radius of the Sun.

Let $R$ denote the radius-vector of the ejected particle at the time $t$.

Let $\varphi$ denote the heliocentric latitude of the point of ejection.

Let $v_{1}$ denote the linear velocity of the particle due to the Sun's rotation.

Let $v_{2}$ denote the velocity of ejection.

Let $V$ denote the resultant initial velocity of the particle.

Let $\psi$ denote the angle between a normal at the point of ejection and the actual initial direction of motion.

The following expressions give the relations existing between the quantities which determine the subsequent motion:

$$
v_{1}=\frac{2 \pi R_{\circ} \cos \varphi}{T_{\circ}}
$$

If $T_{\circ}$ is expressed in seconds, $v_{1}$ is the distance described in one second of time.

$$
\begin{gathered}
V=\sqrt{v_{1}{ }_{1}+v^{2}{ }_{2}} \\
\tan \psi=\frac{v_{1}}{v_{2}}
\end{gathered}
$$

It is evident that so long as the line of action of the ejective force is along a normal, any transverse force, however great or small, will at once determine the position of the plane in which 
the subsequent motion of the particle lies, since the lines of action of both forces must lie in this plane. If there are several transverse forces; the plane of motion must contain a normal through the point of ejection and the resultant of the several transverse forces.

The value of $v_{1}$ is dependent only on $\varphi$, and can therefore be determined accurately. If we assign different values to $v_{2}$ we can evidently compute all the elements of the several orbits which would be described corresponding to different initial velocities, since we also know the mass and radius of the Sun. In other words, we have given the radius-vector and the velocity and direction of motion of a particle at a given instant of time to find all the elements of the orbit described, the central force being known.

Let $\alpha$ denote the semi-major axis of the eclipse described.

Let $K^{2}$ denote the mass of the Sun.

(The value of $K^{2}$ is such that if the Sun acts on a stationary material particle free to move, at a distance equal to the Earth's mean distance from the Sun, the velocity of the particle at the end of one second of time will be equal to $\frac{K^{2}}{24 \times 60 \times 60}$ )

The general equation for undisturbed motion is:

$$
V=K^{2}\left(\frac{1}{R}-\frac{1}{\alpha}\right)
$$

From which we can evidently find $\alpha$.

We next obtain the periodic time $t$ from the familiar expression:

$$
t=\frac{K}{\alpha \frac{3}{2}}
$$

The eccentricity $\varepsilon$ can be found as follows:

Let $R$ and $R^{\prime}$ denote the distances of the particle from the two foci of the eclipse, then for $R=R_{\circ}$ the angle included between $R$ and $R^{\prime}$ is always equal to $180-2 \psi$, so that we can at once write:

$$
\varepsilon=\sqrt{R_{\circ}^{2}+R^{\prime 2}-2 R_{\circ} R^{\prime} \cos \left(180^{\circ}-2 \psi\right)}
$$

from which $\varepsilon$ is readily found.

To determine the inclination of the plane of the orbit to the Sun's equator, we have given a point (the Sun's center) and a line (the actual path of the particle-the projection of this path on the Sun's surface at the instant of ejection being nearly parallel to the equator) through which the plane of the orbit must pass. 
The inclination of the plane must evidently always be nearly the same as the latitude of the point where the particle is ejected.

I have computed the paths of particles for different velocities of ejection in a mean latitude of $15^{\circ}$. The elements are given in the following table:

TABLE I.

\begin{tabular}{|c|c|c|c|c|}
\hline $\begin{array}{l}\text { Initial Velocity } \\
\text { of an Ejected } \\
\text { Particle=V. }\end{array}$ & \multirow{2}{*}{$\begin{array}{l}\text { Angle between } \\
\text { the Normal at } \\
\text { the Point of } \\
\text { Ejection and } \\
\text { the Initial Di- } \\
\text { rection of Mo- } \\
\text { tion }=\psi \text {. }\end{array}$} & \multirow{2}{*}{$\begin{array}{c}\text { Semi-Major Axis } \\
\text { of the Orbit }=\alpha \\
\text { Radius of Sun }=1\end{array}$} & \multirow{2}{*}{$\begin{array}{c}\text { Periodic Time } \\
\text { of the Parti- } \\
\text { cle=t }\end{array}$} & $\varepsilon$ \\
\hline Miles per second. & & & & Eccentricity. \\
\hline $\begin{array}{l}219.1 \\
269.2 \\
311.3 \\
330.3 \\
348.3 \\
357.0 \\
362.1 \\
372.2 \\
377.0 \\
379.5 \\
379.7 \\
382.0\end{array}$ & $\begin{array}{r}0^{\circ} .318 \\
.259 \\
.225 \\
.212 \\
.201 \\
.196 \\
.193 \\
.188 \\
.185 \\
.185 \\
.185 \\
.184\end{array}$ & $\begin{array}{c}0.75 \\
1.00 \\
1.50 \\
2.00 \\
3.00 \\
4.00 \\
5.00 \\
10.00 \\
20.00 \\
40.00 \\
80.00 \\
\text { Infinity }\end{array}$ & $\begin{array}{c}0.075 \\
.116 \\
.214 \\
.329 \\
.604 \\
.930 \\
1.300 \\
3.678 \\
8.263 \\
29.42 \\
83.22 \\
\text { Infinity }\end{array}$ & $\begin{array}{c}0.9933 \\
.9950 \\
.9967 \\
.9975 \\
.9983 \\
.9987 \\
.9989 \\
.9995 \\
.9997 \\
.999+ \\
.999+ \\
1.000\end{array}$ \\
\hline
\end{tabular}

Apparent motions corresponding to component velocities of two hundred and fifty miles per second (the actual velocity is, as a rule, always greater than the observed) are not infrequently observed in solar protuberances. (See Young's General Astronomy, page 208.) It does not, however, follow that the forces producing these motions must be correspondingly great, for if :

$$
\mathrm{V}=\frac{\mathrm{F}}{\mathrm{M}}
$$

(in which $F, V$, and $M$ are respectively the moving force, the maximum velocity generated, and the mass moved), then for a constant force $F$ the velocity $V$ will increase as the mass $M$ diminishes, and as the resistance to motion is to be considered zero, the volume corresponding to a given mass may be either great or small without affecting the velocity.

The above elements show that the theoretical orbit will always be a very elongated ellipse, the perihelion point being near the Sun's center and having a longitude nearly $180^{\circ}$ different from the longitude of the point of ejection, while the nodal points will always be $90^{\circ}$ and $270^{\circ}$ from the theoretical perihelion, and within less than a score of miles of the Sun's center. 
Let us now consider the circumstances of motion of a series of particles ejected from the same point on the Sun's surface following each other so as to form a continuous stream. The angular velocity of each particle as it leaves the Sun's surface will be the same as that of the Sun, but as the radius-vector of the particle increases the angular velocity decreases according to rigorous mechanical laws. Therefore, while each particle may be considered as describing an independent orbit, the curve formed by the stream will not be a conic section, but a helix, which, so long as the latitude of the base of the stream is not zero, will necessarily be of double curvature. As all the particles causing the visible coronal outlines are within a few diameters of the Sun, we can, without appreciable error, consider a given stream as lying in one plane. For simplicity in the discussion, a parabolic velocity will be assumed for all streams:

Let $\omega_{0}$ denote the true anomaly for a radius-vector $R_{0}$ and time $t_{0}$.

Let $\omega$ denote the true anomaly for a radius-vector $R$ and time $t$.

Let $T$ denote time of the theoretical perihelion passage.

Let $t$ denote the time corresponding to $R$ and $\omega$.

Let $\theta$ denote the angle turned through by the Sun during the time $\left(t-t_{0}\right)$.

With the well known relations which exist between $(t-T)$ and $\omega$ in the case of parabolic motion, the results given below are readily obtained. The angle $\theta$ is zero at the instant $t_{0}$ when $\omega=\omega_{0}$ and $R=R_{0}$. Both $\theta$ and $\omega-\omega_{0}$ are measured from the fixed heliocentric direction in space which the origin of the stream has at the instant $t_{0}$. The angle which a line drawn from the base of the stream to any point in the stream makes with a normal through this base, will be called $I$.

TABLE II.

\begin{tabular}{|c|c|c|c|c|c|c|}
\hline $\begin{array}{l}\text { Radius-Vector of } \\
\text { the Particle }=R=\end{array}$ & 1.0 & 2.0 & 3.0. & 4.0 & 5.0 & 6.0. \\
\hline $\begin{array}{l}180^{\circ}-\omega_{0} \\
t-T-T \\
t-t_{0} \\
6000 \\
\theta \\
\theta\end{array}$ & $\begin{array}{c}0^{\circ} 22^{\prime} .1 \\
0^{\mathrm{d}} .009 \\
0^{\mathrm{d}} .000 \\
0^{\prime} .0 \\
0^{\circ} .0\end{array}$ & $\begin{array}{l}0^{\circ} 15^{\prime} .6 \\
0^{\mathrm{d} .025} \\
0^{\mathrm{d}} .016 \\
6^{\prime} .5 \\
0^{\circ} .2 \\
0^{\circ} .2\end{array}$ & $\begin{array}{c}0^{\circ} 12^{\prime} .8 \\
0^{1} .045 \\
0^{\mathrm{d}} .036 \\
9^{\prime} .3 \\
0^{\circ} .5 \\
0^{\circ} .5\end{array}$ & $\begin{array}{l}0^{\circ} 11^{\prime} .0 \\
0^{\mathrm{d}} .070 \\
0^{\mathrm{d}} .061 \\
11^{\prime} .1 \\
0^{\circ} .8 \\
0^{\circ} .9\end{array}$ & $\begin{array}{l}0^{\circ} 9^{\prime} .9 \\
0^{\mathrm{d}} .097 \\
0^{\mathrm{d}} .088 \\
12^{\prime} .2 \\
1^{\circ} .2 \\
1^{\circ} .3\end{array}$ & $\begin{array}{r}0^{\circ} 9^{\prime} .0 \\
0^{\mathrm{d}} .128 \\
0^{\mathrm{d}} .119 \\
13^{\prime} .1 \\
1^{\circ} .6 \\
1^{\circ} .8\end{array}$ \\
\hline
\end{tabular}


A Mechanical Theory of the Solar Corona,

TABLE II-Continued.

\begin{tabular}{|c|c|c|c|c|c|}
\hline $\begin{array}{l}\text { Radius-Vector of the Parti- } \\
\text { cle }=R=\end{array}$ & 7.0. & 8.0 & 10.0 & 20.0 & 40.0 \\
\hline $\begin{array}{l}180^{\circ}-\omega \\
t-T_{0} \\
t-t_{0}-\omega_{0}-10\end{array}$ & $\begin{array}{l}0^{\circ} 8^{\prime} .3 \\
0^{\mathrm{d}} .162 \\
0^{\mathrm{d}} .153 \\
13^{\prime} .7 \\
2^{\circ} .1 \\
2^{\circ} .3\end{array}$ & $\begin{array}{l}0^{\circ} 7^{\prime} .8 \\
0^{\mathrm{d}} .198 \\
0^{\mathrm{d}} .189 \\
14^{\prime} .3 \\
2^{\circ} .6 \\
2^{\circ} .8\end{array}$ & $\begin{array}{c}0^{\circ} 7^{\prime} .0 \\
0^{\mathrm{d}} .278 \\
0^{\mathrm{d}} .269 \\
15^{\prime} .1 \\
\end{array}$ & $\begin{array}{c}0^{\circ} 4^{\prime} .9 \\
0^{d} .782 \\
0^{d} .773 \\
17^{\prime} .2 \\
\end{array}$ & $\begin{array}{r}0^{\circ} 3^{\prime} .5 \\
2^{\mathrm{d}} .18 \\
2^{\mathrm{d}} .17 \\
18^{\prime} .6\end{array}$ \\
\hline
\end{tabular}

The equation which fairly represents the relation between $R$ and $I, I$ find to be the following:

$$
I=\frac{1}{40} R^{2}+\frac{1}{6} R-\frac{1}{6}
$$

$I$ being expressed in degrees of arc and the unit of $R$ being a radius of the Sun.

For discussing the curve represented by the above equation, it will be more convenient to take the origin on the Sun's surface at the base of the stream and refer the curve to a normal through this origin.

For all points in any stream visible during an eclipse we can without sensible error write:

$$
\rho=R-1,
$$

$\rho$ being the distance from any point in the stream to the Sun's surface.

Equation (7) now becomes

$$
I=\frac{1}{40}(\rho+1)^{2}+\frac{1}{6} \rho .
$$

Since for our limits the angle $I$ will always be small, the inclination $I^{\prime}$ of the curve, at any point, to a normal through the origin will be given by the differential expression

$$
I^{\prime}=\rho \frac{d I}{d \rho}+I=\frac{1}{20} \rho(\rho+1)+\frac{1}{6} \rho+I,
$$

$I^{\prime}$ being expressed in degrees of arc.

With the aid of this equation we obtain the following inclinations $I^{\prime}$ for the corresponding values of $\rho$, the values of $I$ being taken from Table II: 
TABLE III.

\begin{tabular}{c|c}
\hline & $I^{\prime}$ \\
\hline 1.0 & $0^{\circ} .5$ \\
2.0 & 1.2 \\
3.0 & 2.0 \\
4.0 & 3.0 \\
5.0 & 4.1 \\
6.0 & 5.4 \\
7.0 & 6.8 \\
\hline
\end{tabular}

If $\beta$ denotes the Earth's angular distance above or below the plane of the Sun's equator at the time of observation, a streamer in latitude $\phi$, in order to be just visible at either pole of the Sun, must have a length $\rho_{\circ}$ given by the equation:

$$
\rho_{\circ}=\frac{R_{\circ}}{\sin (\varphi \pm \beta)}-1
$$

The values of $\rho_{\circ}$ for the two latitudes $15^{\circ}$ and $30^{\circ}$, and for particular values of $\beta$, are given in the following table (referred to the nearer hemisphere):

TABIE IV.

\begin{tabular}{c|c|c}
\hline Latitude on the $\odot=\varphi$. & $\begin{array}{c}\text { Angular Distance of the } \\
\text { Earth above the Plane of } \\
\text { the } \odot \text { 's Equator= } \beta .\end{array}$ & $\begin{array}{c}\text { Length of a Streamer from } \\
\text { the Sun, which will just } \\
\text { be Visible at the } \odot \text { 's } \\
\text { Pole= } \rho_{\circ} .\end{array}$ \\
\hline $\pm 15^{\circ}$ & $\mp 7^{\circ} .25$ & 1.64 \\
\pm 15 & \pm 7.25 & 6.42 \\
\pm 15 & 0. & 2.86 \\
\pm 30 & $\mp 7.25$ & 1.00 \\
\pm 30 & \pm 7.25 & 0.65 \\
\pm 30 & & 1.59 \\
\hline
\end{tabular}

A simple way to represent graphically the apparent changes in the form and position of a streamer, due to the change in the position of the Earth and to the rotation of the Sun, is to construct a model of a single streamer issuing from a sphere and to project the streamer on a plane surface by parallel rays of light. If the sphere be rotated, and the inclination of the axis of rotation varied between the proper limits (corresponding to the positions of the Earth at different seasons of the year), all the peculiarities of the appearances of the coronal streamer can be reproduced and studied at leisure.

In order to show more forcibly the varying form and position 
of a streamer in projection, I have exaggerated the curvature in the model used for obtaining the outline given in Plate VIII.

The streamer represented by a wire rod was made to issue from a latitude of about $15^{\circ}$, and to have such a length that in projection it was just visible at the pole for $\beta=0^{\circ}$. A longer streamer would have necessitated the use of a smaller scale.

The figures at the extremities of the lines representing the streamers correspond to the number of days elapsed since the base of the streamer was on the central meridian of the nearer hemisphere.

Fig. 1, of Plate VIII., corresponds to an inclination $\beta=0$, Fig. 2 to the time when the Earth is near its maximum distance below the plane of the Sun's equator, and Fig. 3 to the time when the Earth is near its maximum distance above the same plane.

The general outlines of the figures on Plate VIII. are evidently the same as would be produced by a series of equidistant streamers issuing from a single latitude only.

If now the streamers issue from different latitudes within the limits of the Sun-spot zones, coronal forms similar to those shown on Plate VI. (which are made from an actual model, as described on the plate itself) will be produced. In a typical corona the mean latitude of the zone from which the streamers issue will always be the same as the latitude of the densest and most extended portion of the coronal outlines. A necessary consequence of the perspective overlapping of these streamers is the production of luminous rays which, in a typical corona, incline away from the adjacent poles, as will be demonstrated further on.

A crucial test of this or any other theory of the corona is afforded by the appearance and behavior of these so called "polar rays." If they really originate at or very near the Sun's polar regions, the laws governing their appearance must be sought for in the physics of the Sun itself. If, however, the present conception of their origin is the true one, they are mainly due to a series of apparent intersections of streamers from the Sun's equatorial region, projected by perspective above or below the poles; that is, the polar rays which we see in any corona have no objective existence. The situation and curvature of these rays and the apparently vacant spaces where such rays seem to be wanting (the so called "rifts") can be accu- 
Plate VIII.
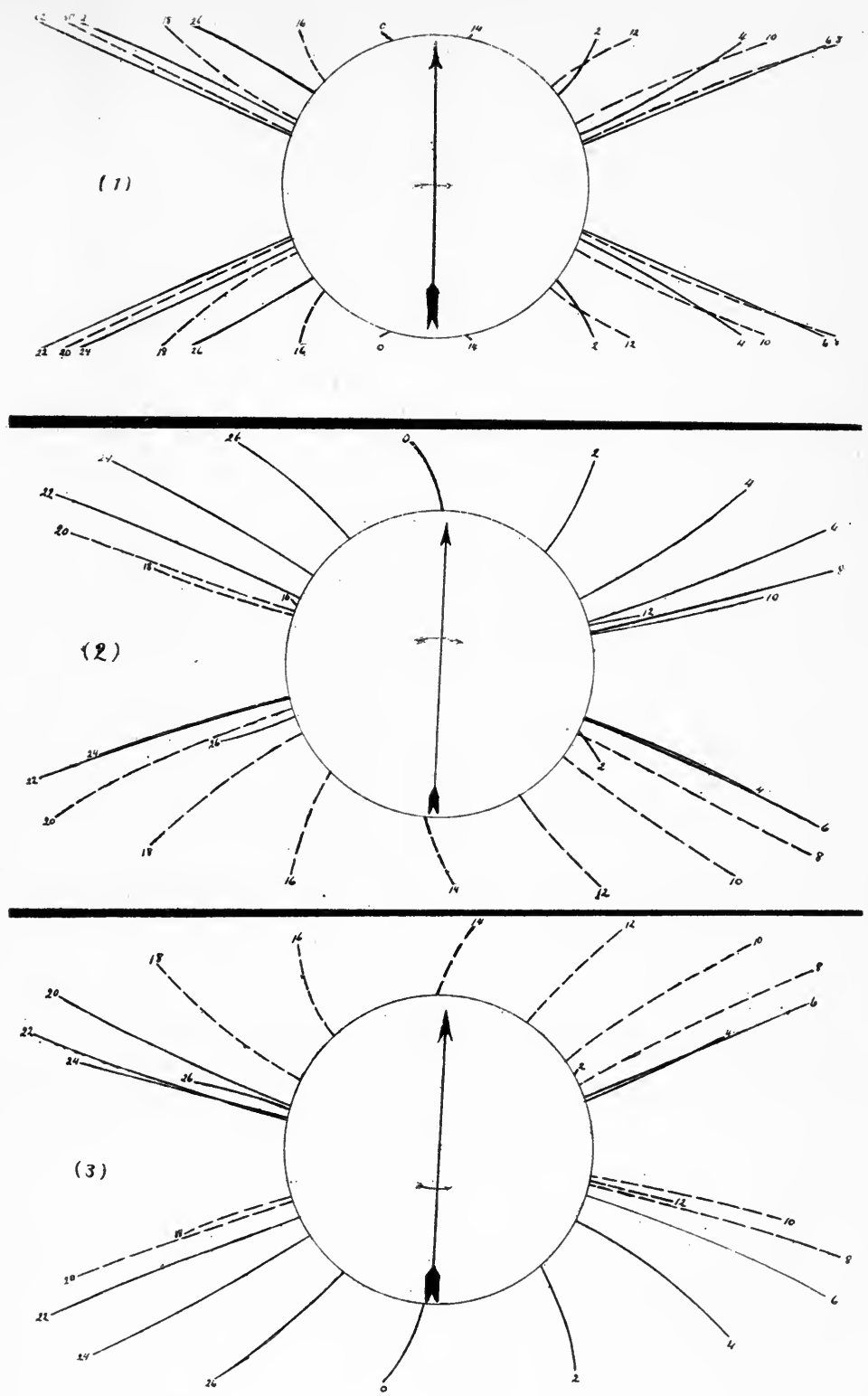

DIAGRAM ILLUSTRATING VARIATION IN THE CURVATURE AND POSITION OF A SINGLE STREAMER IN PROJECTION, BY J. M. SCHAEBERLE. 

rately observed, and the present theory enables one to account for such rays and rifts, and to give the inclinations and general directions of their curvatures.

We will now proceed to investigate the curvatures and situations of the polar rays, first deducing the expressions which give, in projection, the positions and curvatures of the streamers.

I call a "streamer" the actual stream of particles ejected from the Sun. I call a "ray" the subjective appearance produced by overlapping streamers seen in projection, and I write "ray" in italics.

We will first consider the problem for the case when $\beta=0$. Let $\tau$ denote the heliocentric longitude of the base of a streamer in latitude $\varphi$, and let the longitude of the Earth be $\tau_{0}$, the plane of the Sun's equator being taken as the fundamental plane. A normal in latitude $\boldsymbol{\varphi}$ and longitude $\tau-\tau_{0}$ will in projection intersect the Sun's limb at a polar distance $p$ given by the equation:

$$
\tan p=\frac{-\sin \left(\tau-\tau_{\mathrm{o}}\right)}{\tan \varphi}
$$

The position angle $p^{\prime}$ of the inclined stream for moderate polar distances can then be found from the approximate expression:

$$
p^{\prime}=p-\frac{\left[\theta-\left(\omega-\omega_{0}\right)\right] \cos \left(\tau-\tau_{\mathrm{o}}\right)}{\sin \varphi}
$$

The inclination $\left(I^{\prime \prime}\right)$ of the stream to a normal through its base will in projection be given by the approximate expression:

$$
I^{\prime \prime}=\frac{I \cos \left(\tau-\tau_{0}\right)}{\sin \varphi}
$$

Finally, the inclination $I^{\prime}$ to a normal passing through the point at which the inclination is required, will be approximately:

$$
I^{\prime}=I^{\prime \prime}-\left(p-p^{\prime}\right)
$$

Now, owing to the inclinations of the streamers, it is at once evident that even when they are uniformly distributed in longitude, they can never be symmetrically arranged around a radiusvector of the Earth. In projection, however, an approach to a symmetrical form results from a peculiar but well known optical phenomenon.

The streamers which have the least inclination to the line of sight will evidently appear to be most widely separated; that is, they will always be at the areas of minimum density. For the nearer hemisphere the minimum density in projection will 
evidently always be just a little to the west of the Sun's pole, while for the farther hemisphere it will be to the east of the Sun's pole.

Again, as the streamers are slightly curved, those having the least inclination to the line of sight will in projection apparently have the greatest inclination with reference to normals. Hence, when the Earth is near either node, an approximately uniform distribution in longitude of streamers from a given latitude $\phi$ will result in the following arrangement in orthographic projection:

As streamers issuing from the nearer hemisphere have an eastward inclination with reference to normals, the two areas of minimum density will be a little to the west of the Sun's poles; while for the farther hemisphere as the streamers have a westward inclination with reference to normals, the corresponding areas of minimum density will be a little to the east of the Sun's poles.

The inclination to the normal is greatest near the points of minimum density, and this inclination gradually grows less and the density greater as the angular distance from these points increases, until the polar distance $90^{\circ}-\phi$ is reached, where the inclination to the normal is practically zero, and the density at a maximum. (See illustrations of model, Plate VIII.)

The apparent intersections of these streamers produce false streamers, which I have called rays, to distinguish them from the streamers proper.

The form of these rays for a theoretical corona can be determined after the method which will now be considered.

\section{Theory of the Inclined Rays.}

From a series of experiments I have derived the expressions given below, which govern the formation of certain luminous lines (which for want of a specific name I shall simply call rays) produced, as is well known, by the overlapping of two or more sets of alternate bright and dark lines.

If a set of parallel luminous lines, separated by the small angular distance $D$, is crossed at the small angle $J$ by a second set of parallel lines separated by the slightly less angular distance $D^{\prime}$, then when either set is viewed through the other, a new and heavier set of luminous lines (rays) will be formed, which make the angle 
$K$ with the wider set of lines, and the angle $K-J$ with the set of closer lines.

I have deduced the following rigorous expression for the value of $K$ :

$$
\tan K=\frac{D}{D-D^{\prime} \sec J} \tan J
$$

The perpendicular distance $\mathrm{D}_{1}$ between two adjacent lines (rays) of the new set is given by the rigorous expression:

$$
D_{1}=D(\cot J-\cot K) \sin K
$$

-Equation (16) shows that $K$ is always greater than $J$, and reaches a maximum when $D-D^{\prime} \sec J=0$; for this condition $K=90^{\circ}$ and $D_{1}=D \cot J$.

As the angle $J$ increases, the number of intersections to a given surface increases. Now as these intersections cause the phenomenon of the inclined rays (a ray passes through those intersections which are nearest together), it is evident that the rays will be least conspicuous when the number is greatest, since the contrast between different parts of a surface covered by such rays diminishes as the intervals grow smaller.

If one of the two sets of lines is doubled by interpolating a new line midway between each of two adjacent lines, a new ray will be formed midway between each of the original rays.

Similarly, if both sets of lines be supposed to change in the same way, precisely the same effect will be produced, the number of rays being quadrupled.

If this process of bisection is continued indefinitely, a limit is soon reached at which the lines and rays form a practically continuous surface. The inclination of these rays always remains parallel to the original direction corresponding to the particular condition $D-D^{\prime}=x$; the $K$ for this particular series of rays can be distinguished from other series of less conspicuous rays, made by the same set of lines, by writing:

$$
\tan K=\frac{D}{D-2^{\mathrm{n}}\left(\frac{D^{\prime}}{2^{\mathrm{n}}}\right) \text { sec. J }}
$$

The values of $K$ for each of the other sets of inconspicuous rays can be found by substituting in (17'), successively, the values $\frac{D^{\prime}}{2}, \frac{D^{\prime}}{4}, \frac{D^{\prime}}{8} \cdots \cdots \frac{D^{\prime}}{2^{\mathrm{n}}}$, in place of $D^{\prime}$.

The most favorable case for conspicuous rays evidently 
results when $J$ is small, and $D-D^{\prime}=x$ ( $x$ being a small quantity), since for these conditions any two intersecting lines have many points in common, supposing the lines to have sensible breadths, and only one value of $K$ is possible, so that all the rays are inclined in the same direction, thus at once rendering them unmistakable.

Equation (17) shows that so long as $J$ is very small, the number of rays increases very rapidly as $J$ increases. For large values of $J$, therefore, no conspicuous rays will be shown.

As the angle $J=2 I^{\prime}$ will always be small so far as the present investigation is concerned, we can, without appreciable error, write, in place of equation (16), the expression:

$$
\tan K=\frac{D}{D-D^{\prime}} \tan J
$$

I tried to deduce a rigorous expression for the equation of the curve formed by the projection of the nearer set of streamers upon the farther set, but found the conditions too complicated, since there are two sets of curved diverging lines, neither set having a common point of intersection, and the two sets having different envelopes, while the angle of divergence itself is a variable quantity.

$\mathrm{W}$ e can, however, apply the above formula for finding $K$, if we consider only very short portions of the curve, since for very short distances the deviation from parallelism of two adjacent streamers will be small, and consequently the error in $K$ will, as a rule, be small also.

Now for a series of normals, radiating at regular intervals from a small circle whose latitude is $\phi$, the angle between the normals in the nearer and farther hemispheres for the same position-angle $p$ is always zero in projection; hence $K$ is always zero, whatever the relative density of the normals in the two hemispheres may be. The projected intervals will not vary as a function of $p$ alone, since for normals in latitude $\varphi$, the density will be at a maximum when $p=90^{\circ}-\phi$; hence it follows that the intervals vary as some function of $a p, a$ being defined by:

$$
a=\frac{90}{90-\varphi}
$$

Now, in the case of an actual streamer, when the base is in longitude $\tau-\tau_{0}$, the longitude indicated by the direction of the 
stream at a distance $\rho$ from the base will be $\left(\tau-\tau_{\circ}-I^{\prime}\right)$; hence, when the direction of the streams, at a distance $\rho$, corresponds to - $\tau-\tau_{0}=0$ and $\tau-\tau_{\circ}=180^{\circ}$, the bases of these streams will be in longitudes $+I^{\prime}$ and $180^{\circ}+I^{\prime}$, respectively. Now, in projection, the position-angles, $p_{1}$ and $p_{2}$ corresponding to these longitudes, are given by the approximate expressions:

$$
\begin{aligned}
& p_{1}=-\frac{\left[I^{\prime}-I\right]}{\sin \phi}=-I_{1} \\
& p_{2}=+\frac{\left[I^{\prime}-I\right]}{\sin \phi}=+I_{1}
\end{aligned}
$$

In which $I=\theta-\left(\omega-\omega_{0}\right), p$ being measured from the north pole towards the east, through $360^{\circ}$.

$p_{1}$ and $p_{2}$ will be called the secondary coronal poles of the nearer and farther hemispheres, respectively, for the latitude $\varphi$.

The location of the secondary poles can perhaps be better understood from what follows.

At the north pole, for example, streamers from the nearer hemisphere, which are projected on the west side of the secondary pole, appear to radiate from points which are below the Sun's center. As the position angle increases, the center of divergence appears to approach the Sun's center, and then to rise above it, so that streamers on the east side appear in projection to radiate from points above the Sun's center.

Consequently, at the same true polar distances, the density on the east side of the Sun will be greater than it is on the west side. For the streamers of the farther hemisphere projected at the same pole, the arrangement is evidently exactly the reverse.

A streamer is at a secondary pole when its direction in projection is parallel to the Sun's axis.

Equation (16) can be written:

$$
\tan K=\frac{1}{1-\frac{D^{\prime}}{D}} \tan J
$$

From which it appears that $K$ is only dependent on the ratio of $D^{\prime}$ to $D$, and entirely independent of their absolute values.

Let us consider $J$ to be positive when the inclination of the denser set of projected streamers to the less dense set is in the 
direction N.E.S.W., and negative, when the inclination is in the opposite direction; then, in conformity with the law stated on page 58 , the factor:

$$
\frac{D}{D-D^{\prime}}
$$

will be positive in the first and second quadrants (but indeterminate between the limits $p=90^{\circ}-\varphi$ and $\left.90^{\circ}+\varphi\right)$, and negative in the third and fourth quadrants (but indeterminate between the limits $p=270^{\circ}-\varphi$ and $\left.270^{\circ}+\varphi\right)$; hence, according to the above equation, $K$ will always have the same sign as $J$, or, in other words, the rays in latitudes greater than $\phi$ will always be inclined in a direction away from the nearer pole. The angle which the ray makes with a normal will be $K-f \cdot J$, but as $J$ is, as a rule, smaller than the error of the measured inclinations, and $f$ always less than 0.5 , the term $f \cdot J$ can be neglected. $K$ can therefore be considered to be the inclination of the ray to a normal, and if the quadrant is mentioned no attention need be paid to the sign of $K$, as the inclination is in the direction away from the nearer pole.

For finite values the condition $D=D^{\prime}$ will only be fulfilled at the poles where the density is near a minimum; the very short concentric arcs formed by the few intersections of nearly normal streamers inclined to each other at the small angle $J$, will therefore not be visible as a rule. The nearly normal rays at the poles, therefore, nearly coincide with the streamers. This particular phase of the phenomenon is beautifully illustrated by means of the models given in Plate VII., described further on. Figure I. illustrates one of the cases which correspond to the the value $\beta=0^{\circ}$, so that in orthographic projection $D=D^{\prime}$ at the poles; for this exceptional position the slightest variations of the angle $J$, produced either by elevating one set of lines with respect to the other, or by a rotary motion of the plates, causes the ray to swing very rapidly through a large angle, producing forms like Figures II., III., IV., XV., all corresponding to small values of $J$. (Figures X. and XI. represent conditions when $J$ is comparatively large and $D=D^{\prime}$.) Except for critical cases, a given value of $K$ does not change to any great extent for small relative variations of $D, D^{\prime}, J$, as can be seen from an inspection of the figures. At the same position-angle $p$ the value of $K$ will vary but slightly when $p$ is comparatively large, and 
although the apparent changes are very rapid for a given ray, yet as each one assumes a new position another ray is formed which nearly occupies the successive positions of the preceding ray, so that at any given instant the apparent change in the whole system of rays will only result in a lateral displacement of the axis of symmetry, and for the critical cases the concentric rays formed are usually not of sufficient magnitude to be seen, as has been explained above. When both sets of streamers are visible, the pyramidal form of the denser portions of the rays projected at the poles can be regarded as resulting directly from the overlapping of streamers inclined to each other.

Now the streamers at the nearer secondary pole are projected against streamers of the farther hemisphere, which are at an angular distance of $2 I(=J)$ from the farther secondary pole; for the polar distance $p$ the ratio of the intervals $D$ and $D^{\prime}$ is therefore a function of $a p$ and $I^{\prime}$. This function must be so determined that the density is least, but not zero, when $p=0 \pm I^{\prime}$, and a maximum finite density when $p=90^{\circ}-\phi$, or $a p=90^{\circ}$.

Let $\delta$ and $\delta^{\prime}$ denote the densities of the two sets of streamers for a given polar distance $p$. We then have as a rough approximation for values of $p$ greater than $I$ :

$$
\begin{aligned}
& \delta=d \sin \left(a p+I_{1}\right) \\
& \delta^{\prime}=d \sin \left(a p-I_{1}\right)
\end{aligned}
$$

The value of $d$ being determined by the condition:

$$
d=\frac{\delta_{0}}{\sin I_{1}}
$$

In which $\delta_{0}$ is the density at the true pole.

In place of the density $\delta$, we can, for moderate polar distances, substitute the reciprocal of the corresponding interval $D$, and write:

$$
\delta=\frac{1}{D} \quad \delta^{\prime}=\frac{1}{D^{\prime}}
$$

Hence:

$$
\frac{D^{\prime}}{D}=\frac{\delta}{\delta^{\prime}}=\frac{\sin \left(a p+I_{1}\right)}{\sin \left(a p-I_{1}\right)} .
$$

Substituting this value of the ratio of the intervals in equation (21), and reducing, we obtain, finally:

$$
\tan K=\frac{\sin (a p-I)}{2 \cos (a p) \sin I} \tan 2 I^{\prime}
$$


$2 I^{\prime}$ being substituted in place of $J$. As $I^{\prime}$ is small we can write $\tan 2 I^{\prime}=2 \tan I^{\prime}$; hence,

$$
\tan K=\frac{\sin \left(a p-I_{1}\right)}{\cos (a p) \sin I_{1}} \tan I^{\prime}
$$

The ratio of $\tan I^{\prime}$ to $\sin I_{1}$ will be practically constant for moderate values of $p$. When $a p=90^{\circ}$, equation (27) becomes indeterminate. If the streamers were of infinitesimal breadth in latitude, they would coincide with the rays when $p=90^{\circ}-\phi$. In reality, however, the extension in latitude may be considerable, and the effect of this extension is to make a practically continuous surface of rays before the polar distance is as great as $90^{\circ}-\varphi$. Hence, polar rays at considerable polar distances will usually be lost in the general illumination.

From the preceding discussion it follows that for the case of $\beta=0^{\circ}$ the axis of the typical corona will, in orthographic projection, coincide with the Sun's axis.

Changes in the Form of the Typical Corona Resulting from Variations in the Heliocentric Latitude of the EARTh.

When the Earth is above $(\beta+)$ or below $(\beta-)$ the plane of the Sun's equator, the quantities $D, D^{\prime}$, and $J$ undergo certain changes for the same value of $p$ in projection.

When the Earth is below the plane of the equator, the nearer streamers of the northern hemisphere will appear more dense, with a slightly less inclination to the normal than is the case when $\beta=0$, while the density of the streamers from the farther hemisphere will be diminished; but the increase in the westward inclination to the normal will be greater than the diminution of the eastward inclination in the nearer hemisphere. $\checkmark$ has therefore increased, but this increase is, in part, at least, counteracted by the decrease in the value of the factor $\frac{D}{D-D^{\prime}}$.

With reference to the streamers, therefore, $K$ does not necessarily change to any great extent. But as the nearer streamers have a less eastward inclination, while the farther ones have a greater westward inclination, the whole system of rays formed will, according to the fundamental principle laid down in the law given on page 58, have a greater eastward inclination. The 
effect of this common change in the inclination of the rays is to cause an apparent shifting of the coronal pole towards the WEST.

For the same reason the south coronal pole will be shifted towards the EAST.

When the Earth is above the plane of the equator, the north pole of the corona will be on the east side of the projected position of the Sun's pole, and the south coronal pole will lie on the west side.

As the projections seen from the Earth are not strictly orthographic, the parallactic effect has a constant tendency to throw both coronal poles slightly towards the east of the position they would occupy if viewed from an infinite distance.

Changes in the form of the typical outer corona, due to variations in $\beta$, for streamers of a given magnitude and distribution, can be readily understood, with the aid of the prints and diagrams given in Plates VI. and VIII., in connection with Tables IV. and V., and also from what follows further on.

A want of uniformity in the distribution of the streamers in longitudes $\tau-\tau_{\circ}= \pm 0$, or $180^{\circ}$, may result in the partial or total destruction of the symmetrical inclined polar rays, and in other longitudes the same irregularities will cause a deformation of the general typical features of the corona. An unsymmetrical corona is, therefore, in no way to be regarded as contrary to the theory.

If, for any cause, there is real or apparent periodic variation in the mean latitude of the streamers, the ratio of the polar to the equatorial extension will, of course, be subject to a similar variation. (See postșcript.)

From Table IV., we see that for two streamers in latitude $15^{\circ}$ and $30^{\circ}$, respectively, just visible at the poles in projection, the one in latitude $30^{\circ}$ will have only about one third of the equatorial extension that is shown by streamers in $15^{\circ}$ latitude. If, therefore, the mean latitude of greatest solar activity is apparently shifted towards the higher regions of the Sun-spot zones, the coronal outlines may become practically circular. The criterion for determining from the observed outlines whether $\beta$ is large or small will no longer be satisfactory, since the ratio of the polar to the equatorial extension now undergoes but a slight change for all possible values of $\beta$. However, when the polar rays, or the wing boundaries adjacent to the poles, 
are present, the position of the axis of the inner corona will, as a rule, at once tell whether the observer was above, below, or in the plane of the Sun's equator. The inclinations of the boundaries of the wings, at the Sun's outline, can be used to indicate, approximately, the position of the pole of the inner corona when the rays are wanting.

Now, when the Earth is at considerable distances above or below the plane of the Sun's equator, and the equatorial streamers are visible for only a moderate distance, the more depressed set may show so faintly that they apparently fall short of reaching the pole in projection. For this case, equation (16) shows that $K=0$, since $2 I^{\prime}$ is then zero; so that if all the streamers were confined to a single circle of latitude and only one set visible at the pole, the true projections of the streamers would be seen, and the direction of curvature would always be governed by rigorous conditions. (See Plate VIII.)

For this case, when the symmetrical inclined rays are present, the phenomenon admits of the same simple explanation, which accounts for the polar rays when $\beta$ is nearly zero, since streamers on opposite sides of the Sun, whose latitudes differ by $2 \beta$, will, in projection at the poles, produce a similar arrangement of the rays; but these rays will not necessarily extend to the outer limit of the coronal structure at the poles, and for this reason they may not be conspicuous when $\beta$ is large.

When, however, the polar rays are very conspicuous and uniform for large values of $\beta$, and a small equatorial extension, the phenomenon is still to be accounted for in the same general way, which we will now consider.

As the curvature and deviation from the normal depend in projection upon the latitude $\varphi$, streamers in different latitudes with nearly the same longitude will, in projection, apparently intersect each other, and consequently curved rays or outlines will be formed, and these may be wholly produced in either the nearer or farther set of streamers. For the same position-angle $p$, streamers in latitude $\phi^{\prime}$ will not appear to radiate from the same points that those in latitude $\varphi$ do. If the secondary poles for latitude $\varphi^{\prime}$ are given by the expressions:

$$
\begin{aligned}
& p_{1}{ }^{\prime}=-I_{1}{ }^{\prime} \text { nearer hemisphere, } \\
& p_{2}{ }^{\prime}=+I_{1}^{\prime} \text { farther hemisphere, }
\end{aligned}
$$

Then for $+\phi^{\prime}$ greater than $+\phi$ we would always have $I_{1}^{\prime}$ less than $I_{1}$. This results directly from the fact that the change in 
the directions of the streams varies nearly with $\rho^{2}$, and in projection at the poles the ratio of $\rho$ to $\rho^{\prime}$ is nearly the same as the ratio of $\operatorname{cosec} \phi$ to $\operatorname{cosec} \phi^{\prime}$. The streamers in latitude $\phi$ will therefore be more inclined to the normal than those in latitude $\phi^{\prime}$. In projection, therefore, streamers on the east side of the secondary pole in latitude $\phi$ will appear to radiate from points which are nearer than the corresponding points for latitude $\phi^{\prime}$, while on the west side of the Sun the points on the osculating curves are at greater distances for latitude $\phi$ than they are for the latitude $\phi^{\prime}$. The resulting apparent variation in density will therefore produce the same phenomenon which results from the projection of the streamers of the nearer hemisphere upon those of the farther. Inclined polar rays may therefore be formed by the overlapping of streamers having nearly the same longitude but different latitudes. For this case the $I_{1}$ of equation (24) would be the angular distance between the secondary poles for latitude $\phi$ and $\phi^{\prime}$, while $I^{\prime}$ would correspond to the difference of the projected inclinations at the pole for the given latitudes.

If we assume the ratio of the latter to the former to be $c$, and neglect the small angle $\left(I_{1}-I_{1}{ }^{\prime}\right)$, equation (27) reduces to:

$$
\tan K=c \tan \left(a p^{\prime}\right)
$$

In which $p^{\prime}$ is now the distance from the mean secondary pole. This system of rays will be more probable for large values of $\beta$, and will not be symmetrical with reference to the poles of the Sun. The inclinations will vary with the value of $c$; these variations will, however, as a rule, be small, since the values of $\beta, \phi$, and $\phi^{\prime}$ are subject to comparatively small variations.

When $\beta$ is zero, the streams of the nearer hemisphere projected against those of the farther hemisphere will produce a corona whose axis will be practically coincident or parallel with the Sun's axis. The density (in projection) at the poles may at times be so great that only the wings are seen; the boundary of these wings, even at the poles, will then extend to a limit at which the polar rays begin.

Equation (21) shows that the inclination of the rays is independent of the magnitude of the intervals, so that even when the density is very great or very small, the inclination $K$ of the polar rays, when these are present, will, for a given value of $\beta$, always be practically the same for a uniform distribution of the streamers. 
The four great curves which bound the outer portions of the wing-like coronal outlines, which form a prominent feature of so many eclipses, are the envelopes of the outer portions of the streamers, the density at points on the envelopes being such that the intervals are just filled out. The positions of these envelopes are largely determined by the projection of the more depressed set of streamers against the more elevated set, or vice versa. (See Plate VI.)

To obtain an approximate analytical expression for these envelopes, let $\gamma_{1}^{\prime}$ be the distance from the Sun's center to the point on the Sun's axis, where one set of streamers in latitude $\varphi$, produced backward, would, in projection, just form a continuous surface, and let $P^{\prime}$ be the polar distance of the point where the boundary of this continuous surface cuts the limb of the Sun in projection. Then the distance $\gamma^{\prime}$, at which the intervals between adjacent streamers will just be filled out for different values of $p$, will be approximately given by the expression:

$$
\gamma^{\prime}=\gamma_{1}^{\prime} \sec (a p)
$$

In which $\gamma_{1}^{\prime}$ is a function of the actual density, and equal to the distance from the Sun's center to the point where the envelope proper, or the envelope produced, cuts the Sun's axis.

For values of $\gamma_{1}^{\prime}$, less than the Sur's radius, the approximate expression (31) will answer:

$$
\gamma_{1}^{\prime}=\frac{1}{\sec \left(a P^{\prime \prime}\right)}
$$

For the envelope of the other set of streamers, projected at the same pole, we have:

$$
\begin{gathered}
\gamma^{\prime \prime}=\gamma_{2}^{\prime \prime} \sec (a p) \\
\gamma_{2}^{\prime \prime}=\frac{1}{\sec \left(a P^{\prime \prime}\right)}
\end{gathered}
$$

Similarly, streamers in latitude $\phi^{\prime}, \phi^{\prime \prime}$, etc., will have envelopes of the same general character. The overlapping of all these envelopes finally produces the outlines of the four great wings.

The principal boundary curve or curves will be approximately represented by the equation:

$$
\gamma=\gamma_{1} \sec (a p)
$$

The value of $\gamma_{1}$ being found from data taken directly from the photographs. 
Equation (34) is the locus of a curve of indefinite extent, which leaves the Sun's surface at the polar distance $P$, or cuts the Sun's axis at a distance $\gamma_{1}$ from the Sun's center, the inclination to. the normal varying from $90^{\circ}$ for $p=0$ to $0^{\circ}$ for $p=90^{\circ}-\phi$. The curve has for its asymptote a produced diameter of the Sun in a position-angle nearly equal to $90^{\circ}-\phi$, in which $\phi_{\mathrm{m}}$ is the mean latitude of the streamers.

If the distribution of the streamers in longitude is not uniform, equation (34) will not necessarily represent the observed form for small values of $p$, and if the terrestrial atmospheric conditions are unfavorable for showing the outer corona, the same equation will apparently fail when $p$ is large.

The inner boundaries of these wings will be determined by similar conditions. As the forces at the equator are presumably less active than those having a small latitude, the envelope of these portions of the projected streams will in general be concave near the Sun's disk. The closing of the wings (or fish tails as they are sometimes called) and the decrease in density of the exterior boundaries, result directly from a gradual increase in the value of $\beta$.

The trumpet-shaped outline of the more distant portions of the wings which were first photographed in January, 1889, is theoretically just what is called for. Since the brightness of the streamers decreases with increasing distances from the Sun's surface, the illumination can, by contrast, only be of indefinite extent in those directions where many of these streamers overlap each other, that is, in position-angles corresponding to $\pm\left(90^{\circ}-\phi\right)$ and $\pm\left(270^{\circ}-\phi\right)$. The equation of the outer boundary curve is nearly of the form expressed by formula (34).

The figures given in Plate VI. would be still more strikingly similar to coronal outlines were it practicable to represent the decrease in the brightness of the streamers (represented by the needles in the model) with increasing distances from the center.

\section{Polar Gaps or Rifts.}

There are several different causes which unite to produce an apparent extinction of the polar rays for increasing polar distances. The first of these is made evident from the following considerations: Viewed from a given point, a series of equally 
bright radial lines (and practically equidistant from the observer) of indefinite extent will not appear equally bright to the eye. The lines which are less inclined to the visual rays will always be brighter than the more inclined ones, since in a given direction the density of the luminous particles which make up the line increases for decreasing inclinations. Hence, at a given actual distance from the Sun, a streamer will appear brightest when it is projected at the poles.

A second reason why the rays at the pole are the brightest is the following: Beams from two sources of light of the same absolute quantity, but of different angular magnitude, will be affected differently in passing through an absorbing medium like our atmosphere. If in one case the light is concentrated in a series of points or lines in a given surface, and in the other case the same amount of light is distributed over the whole of the same surface, the absorbing effect of our atmosphere, through which the two sets of luminous beams pass, will be more marked in the latter case than it will in the former. Referring now to the illustrations given in Plate VII., it will be noticed that the number of rays increases with the inclination, while the magnitude of these same rays decreases with the inclination.

(In order that the outer extremity of an inclined ray shall be at as great a distance from the Sun's limb as a ray at the pole, the ratio of the length of the former to the length of the latter must be nearly as sec $K$ to unity; or, for the same length of inclined and polar rays, the corresponding ratio of distances from the Sun's limb would be nearly as $\cos K$ to unity.) The absorbing effect of our atmosphere will therefore always have the tendency to cause a greater weakening of the more inclined rays up to the position where the increase in density suddenly rises in projection (in the model this absorptive effect is of course not included, hence the gaps are not strongly shown), the effect of which is to cause a great contrast between the comparatively bright boundaries of the wings and the more conspicuous polar rays. An inspection of the best photographs, however, will show that these spaces, apparently devoid of dense coronal matter, are often really filled out with faint rays, which in a poor photograph would not show at all. The existence of rifts in other positions is illustrated in the models. When the density of the streamers is so small that no definite 
boundary of a wing is formed, the rays may be visible to a polar distance nearly equal to $90^{\circ}-\phi$. Even if the streamers are not of a gaseous character, their boundaries will not be sharply defined, since the variation in latitude results in intersections at very small angles; any attempt, therefore, to follow the course of any particular one of these streamers, projected as it is against many others, may be wholly in vain.

\section{Graphical Representation of Polar RAYS.}

In the model (Plate VI.) the diameter of the needles compared with the diameter of the ball is too great to admit of the use of a sufficient number to produce the phenomenon of inclined rays satisfactorily.

I have, however, been able to fulfill the conditions imposed by a modification of the graphical method. In projection, the streamers near the poles will appear to be tangent to a small circle concentric with the Sun, instead of passing through the Sun's center. Accordingly, instead of using slightly inclined needles radiating from the surface of a sphere, $I$ formed a series of alternate bright and dark lines on an opaque film on the surface of a piece of plate glass. These radial lines (slightly curved) were all drawn tangent to the same small circle, so that for a given distance from the center of this circle the inclination to a normal was approximately the same as required by theory. On this plate a second plate copied from the first was laid, in both the direct and reversed positions.

Now the condition which must be fulfilled is this: The lines of one plate must all be inclined in the same direction with reference to the lines of the other plate, and this arrangement must still produce rays which incline away from the lines representing the secondary poles, if the theory is true. While I had no doubt, from theoretical considerations, as to what the pictorial result of the combination of the two gratings would be, I had hardly hoped to obtain such satisfactory results on a first trial. In Plate VII., Figures I., II., III., IV., VIII., X., XI., $\mathrm{XV}$., $a$ and $c$ illustrate some of the many, forms which can be produced by streamers having nearly the same longitude, but different latitudes, the plates for this purpose being superposed without reversing. The remaining prints V., VI., VII., IX., 
XI., XII., XIII., $b, d$, and $e$, illustrate some of the forms produced when the streamers of the nearer hemisphere are projected against those of the farther hemisphere, the plates being reversed for this purpose. The shifting of the secondary coronal pole is also represented (see Figures VIII. and IX., Plate VII.); one set of lines being slightly raised or lowered to correspond to a change in the position of the Sun's axis, which, in projection, is supposed to be parallel to the edge of the paper.

The original prints were made on platinum paper, the two superposed gratings representing the negative. These prints, like those of Plate VI., were then fastened to a large sheet and photographed so as to obtain on a single page all the different forms shown in Plate VII.

If a set of alternate bright and dark straight lines radiating from the same point is placed upon another set of lines also radiating from a point, no curves will be formed so long as the centers of divergence are in the same line of sight; but the instant one center is shifted with reference to the other, curves quite similar to those given in Plate VII. will be produced. If the plates are separated from each other, the errors in the directions and positions of the lines will have a much less distorting effect upon the rays than when the plates are close together.

For the purpose of making the prints, however, it was necessary to place the plates close to the platinum paper; all the irregularities are therefore shown in these prints. It must be remembered that from the nature of the model the outer portions of the rays shown in the figures of Plate VII. are much too bright and prominent, since the intensity does not diminish with the distance from the center of divergence, as it evidently does for the actual corona.

Then, again, in the model the contrast between the bright and dark lines is abrupt and sharp, so that in the prints, for a near view, these lines show plainly with the rays; but in the case of actual streamers this state of things would evidently not exist. If the plate is removed several feet from the eye, only the rays will be distinguishable to the unaided vision.

Note.-If each eruption is regarded as resulting in a large number of slightly diverging pencils of matter, two eruptions on the central meridian in slightly different latitudes would, in projection at the poles, also cause the phenomenon of inclined rays. 
If several plates are superposed, the contrasts are strengthened, and grotesque figures, resulting from irregularities in the gratings, are not uncommon.

The boundaries of the wings in the hemisphere which contains the visible pole will, even for comparatively small values of $\beta$, usually appear double or multiple on the side turned toward the adjacent pole, as portions of both sets of streamers can be seen directly.

When $\beta$ is exactly zero, and the density of the streamers uniform and great, the outer boundary of the wings, even at the poles, may inclose the area usually occupied by the polar rays. The density of the coronal areas will then increase toward those axes whose latitudes are the same as the mean latitudes of the streamers. The outlines of the fainter and more distant portions, rendered visible by the overlapping of many faint streamers projected along these axes, will then be approximately represented by equation (34). If, however, for any cause the streamers from the low latitudes are not decidedly more conspicuous than those in higher latitudes, or if there is a real or an apparent periodic increase in the latitudes of the streams, which at times transfers the scene of greatest visible activity to the higher limits of the spot-zones, the outline of the bounding curves of the wings is more likely to form a closed figure, and the general coronal boundary will be more nearly circular, since the contrast between the areas of different brightness for increasing distances may not be sufficient to bring out the envelope whose approximate equation has already been considered.

From what has already been said, it is evident that the condition of the Earth's atmosphere at the place and time of observation, determines, in a very great degree, the form of the visible buundary of the outer corona.

Before giving further results of the comparison between theory and observation, it is proper to make a few remarks relating to the difficulty under which one labors in making an unbiased examination of published data which are believed to be representations of a certain theoretical form.

One is very apt to overestimate the importance of certain coincidences and to disregard as unimportant certain discrepancies. For instance, some of the evidence tending to prove that the streamers issue from the Sun's equatorial regions 
depends to a certain extent upon the observed variations of the general outlines of the outer corona for different values of $\beta$. So far as this particular evidence is concerned the conclusion reached must depend largely upon the data derived from mere drawings, as the number of reliable photographs secured, for different eclipses, is hardly sufficient to decide the case definitely. The interpretation of these drawings and poorer photographs, by different unbiased observers, will not necessarily be the same.

Certain features should, however, always be present in good photographs, giving evidence tending to prove that the streamers originate in the spot-zones; of these the equatorial outlines of the outer corona are perhaps the most important. The variation in the position of the secondary coronal poles, according to the position of the observer with reference to the Sun's equator, also gives decisive evidence on this point.

For those eclipses of which we have photographs showing polar rays suitable for measurement, I have used the constants for a mean latitude of $15^{\circ}$ for the streamers, and placed $\beta=0$ for finding $K$. As the exact nature of the distribution of the streamers in latitude is not known, any attempt to take into consideration the slight variations in $K$, due to variations of $\beta$ between $0^{\circ}$ and $8^{\circ}$, in order to obtain a closer agreement between theory and observation, would seem to be superfluous. For those photographs of eclipses in which the rays are not shown, I have used the following method for obtaining the direction of inclination of the axis of the inner corona with reference to the Sun's axis. At the origin of the curves bounding the wings adjacent to the poles, tangent lines were drawn. Through the two points of intersection, formed by the two lines near each pole, the coronal axis is supposed to pass; when this line does not pass through the Sun's center, I have assumed it to be parallel to the coronal axis, as the parallactic effect in a typical form is such that both poles of the corona are shifted towards the east of the points they would occupy in orthographic projection. The data for finding the position of the Sun's center with reference to the center of the Moon is, also, nearly always wanting.

As this shifting of the secondary poles will ordinarily be only a few degrees, I have preferred to refer all the measures 
to the true solar poles, rather than to the coronal poles; this treatment, when $\beta$ is large, will of course make the differences between computation and observation larger than they would be if the coronal poles were taken as the origin.

For the above named conditions we therefore have for the neighborhood of the poles and at the Sun's limb the following numerical values:

Hence,

$$
\frac{\tan I^{\prime}}{\sin I_{1}}=c=1.5, a=1.2, \text { and } I_{1}=2^{\circ} ;
$$

$$
\tan K=\frac{1.5 \sin \left(1.2 p-2^{\circ}\right)}{\cos (1.2 p)}
$$

The following are the numerical values of $K$ for each $10^{\circ}$ of distance from the adjacent pole:

\begin{tabular}{c|c|c|c|c}
\hline$p$ & $10^{\circ}$ & $20^{\circ}$ & $30^{\circ}$ & $40^{\circ}$ \\
\hline$K$ & $15^{\circ}$ & $31^{\circ}$ & $46^{\circ}$ & $58^{\circ}$ \\
\hline
\end{tabular}

With reference to the form of the outer corona, as shown by any particular photograph, a very great deal depends upon the mode of development of the photographic plate. A corona with great equatorial extension can be made to appear nearly circular, especially if the atmospheric conditions at the time of an eclipse are poor, so that only the brighter parts are shown. In dealing with drawings not made from photographs, a rude approximation to the true general outline can, at best, only be obtained by combining the results of many different observers of the same eclipse. Such drawings are of little or no value for determining the sign of $\beta$. The individual results of pure drawings are often so discordant among themselves that it is difficult to comprehend how they can possibly be reconciled so as to represent the same phenomenon.

Bearing in mind the fact that in the graphical representation the intensity or distinctness of each streamer does not decrease as the distance from the center increases, the prints from the model, of which illustrations are given in Plate VI., can, in a general way, be used to represent typical coronas. The outline of the outer corona gives a rough indication of the amount of the inclination of the Sun's axis; while the indi- 
cated positions of the poles of the inner corona at once determine whether the observer is above or below the plane of the equator. It will be noticed that even in this simple model the boundaries of the wings indicate the positions of the coronal poles. The reason why the polar rays are not satisfactorily formed has already been stated.

TABLE V. (Plate VI.)

\begin{tabular}{c|c}
\hline No. of Figure. & Corresponds to Season of the Year. \\
\hline & \\
\hline 1 &
\end{tabular}

Observations of Solar Eclipses and Comparisons with THEORY.*

Eclipse of 1715, May 2.

The individual drawings and descriptions are discordant among themselves.

$$
\text { 1776, February } 9 .
$$

Same remarks as above.

$$
\text { 1806, June } 16 .
$$

The drawing is described as being a mere diagram, showing the general effect of the light about the dark Moon. Equatorial streamers evidently not markedly large. (Maximum of spots, 1804; minimum, 1811.)

$$
\text { 1842, July } 8 \text {. }
$$

Imperfect records. The observers supposed the equatorial extension to be the zodiacal light. Equatorial extension great. (Maximum of spots, 1837; minimum, 1843.)

* All the references are to A. C. RANYARD's work " Observations made during Solar Eclipses," Mem. R. A. S., Vol. XLI., unless otherwise stated. To make possible a comparison between the variations in the general details of the corona, as influenced by the phenomena of outgoing streamers retarded by incoming ones (see postscript), I have added the times of maxima and minima of Sun spots, as determined by the investigations of Schwabe, Wolf, and others. 


\section{1, July 28.}

The daguerreotype taken at Königsberg shows only the inner and brighter portions of the corona. The resemblance to the inner corona of July, 1878, is apparent. As required by the theory for a typical form, when $\beta$ is positive, the axis of the inner corona is inclined eastward, according to the inclination of the faint boundaries of the wings just visible. (Maximum of spots, 1848; minimum, 1856.)

\section{3, November 30.}

RANYARD gives no drawing of this eclipse, but the description is just what the theory requires it to be when the Earth is near the node. (Five years after maximum, and three years before minimum of spots.)

1858, September 7.

The drawing in general agrees with the form required by theory when $\beta$ is large. (Compare with Figure 7, Plate I.) (Minimum of spots, 1856; maximum, 1860.)

\section{0, July 18.}

The photograph by Monserrat shows only the innermost parts of the corona. The faint traces of the wing boundaries indicate that the inner coronal axis is inclined towards the east, as it should be. The drawings are very discordant among themselves, and show much polar extension. The majority of the drawings also give an eastward inclination to the coronal axis. Many of the drawings, especially the grotesque one by TempeL, I can reproduce almost exactly, with a model. (Maximum of spots, 1860.)

\section{7, August 18.}

No photographs; the one drawing, which does not agree with the description by the observer, shows great equatorial extension, with only a few short rays at the pole. (Minimum of spots, 1867.5.)

\section{8, August 18.}

All the drawings agree in giving evidence of a considerable inclination of the Sun's axis. (Compare the drawings with the denser portions of Figure 5, Plate I.) The majority of the drawings give evidence of an eastward inclination of the inner 
coronal axis corresponding to a positive value of $\beta$. (Minimum of spots, 1867.5; maximum, 1870.5.)

\section{9, August 7.}

Both the photograph and the drawing are in accord with the theory. In the drawing of MEEK and Sснотт the extension in the equatorial region is greatest at the equator, while the general outlines, both in the photograph and drawing, are quite similar to the inner portions of Figure 5, Plate I. According to the boundaries of the wings as shown on WhIPPLE's photograph, the axis of the inner corona is inclined eastward as required by theory when $\beta$ is positive. (Minimum of spots, 1867.5; maximum, 1870.5.)

\section{0, December 22.}

The Willard photograph of this eclipse, aside from the fact that the polar rays and other fine details of structure are wholly wanting, is strikingly similar to our Cayenne photographs, when they are printed so as to show only the same extent. No fine coronal details are shown. On the Brothers photograph the Sun seems to have reappeared during the exposure. The various drawings of the outer corona are discordant among themselves. As required by theory, when $\beta$ is negative, the wing boundaries indicate that the axis of the inner corona is inclined towards the west. (Maximum of spots, 1870.5.)

\section{1, December 12.}

The four excellent drawings by DAwson, give a quadrilateral outline to the outer corona, but the others do not as a rule give evidence of a small value for $\beta$. The photographs taken at Baikul and Dodabetta, however, give almost exactly the same detail. Practically the same structure is shown at both poles, so that a superficial inspection of the photographs alone will not determine which pole is turned towards the Earth. The boundaries of the wings extend quite up to the poles, so that only the nearly normal rays at the poles are shown on the photographs. At the time of this eclipse, the Earth was nearer to the plane of the Sun's equator than it was at any previous or subsequent eclipse of which photographs were obtained. Compared with the equatorial extension, the polar extent was very 
large. The observed general outline can, however, be fully satisfied by supposing the eruptive forces to have been apparently more active near the superior limits of the Sun-spot zone, or that the equatorial streamers suffered retardation by coming into contact with returning streams. As the original negatives were only three tenths of an inch in diameter, considerable uncertainty may exist in the fainter outlines of the enlarged prints: I was so fortunate as to have the use of a good positive, kindly loaned to me by Mrs. R. A. Proctor (evidently made from a copy of the original negative and enlarged about three diameters). I made a number of shorter timed negatives, and these give, near the Sun, greater excess of equatorial extent and less of polar extent than a positive of the January 1, 1889, eclipse treated in the same way, but the boundaries are not as sharply defined in the former case as they are in the latter. From experiments with a model, the forms of the more luminous confused masses are so plainly the result of apparent intersections of streamers (some of which are doubtless descending) that a more detailed comparison can be dispensed with, since no known forces could possibly account for the various forms which can be traced on this photograph, if we suppose the observed outlines to represent actual forms in space. As required by the theory, the axis of the inner corona is nearly coincident with the Sun's axis, being slightly inclined towards the west, $\beta$ having a negative value. (Maximum of spots, 1870.5; minimum, 1879.0.)

\section{4, April 16.}

There were no photographs taken at this eclipse. It is described as being similar to the eclipse of August, 1868. As drawn by STONE, the axis of the inner corona, given by RANYARD, is inclined towards the west, in agreement with the typical form. A mere drawing, however, is deserving of but little weight so far as the location of this axis is concerned.*

$$
\text { 1875, April } 6 .
$$

This eclipse is described as being curiously similar to the one of the previous year (April 16). The photographs by LOCKYER

*Note.-The observed data for comparison between theory and observation, up to and including this date, have been taken from RANYARD's excellent work on Solar Eclipses. 
and Schuster give unmistakable evidence of a considerable inclination of the Sun's axis, as shown by the boundaries of the wings. The telescopes were not driven by clockwork, so that the polar rays are not shown. The direction of the Sun's axis is not indicated with the necessary accuracy to determine the inclination of the inner coronal axis with certainty. (Maximum spots, 1870.5; minimum, 1879.0.)

$$
\text { 1878, July } 29 .
$$

The first available photographs on which the finer details of the structure at the poles are of such a character as to permit of their being used to test the correctness of the theory were obtained at this eclipse. In the drawing, made by Professor Harkness, from the photographs obtained at this eclipse, published in the volume issued by the United States Naval Observatory, the polar streamers are so well defined that I have been able to make the following comparison between the observed $(0)$ and computed $(c)$ values of $K$. As the measures are necessarily only approximate, I have recorded the measured inclinations to the nearest five degrees for each ten degrees of polar distance available:

18\%8, July 29-Observed and Computed Values of $\boldsymbol{K}$.

\begin{tabular}{|c|c|c|c|c|c|c|c|c|c|c|c|c|}
\hline \multirow{3}{*}{ QUADRANT. } & \multicolumn{12}{|c|}{ Distances from Nearer Pole. } \\
\hline & \multicolumn{3}{|c|}{$10^{\circ}$} & \multicolumn{3}{|c|}{$20^{\circ}$} & \multicolumn{3}{|c|}{$30^{\circ}$} & \multicolumn{3}{|c|}{$40^{\circ}$} \\
\hline & $o$ & $c$ & $0-c$ & $o$ & $c$ & $o-c$ & $o$ & $c$ & $o-c$ & $o$ & $c$ & $0-c$ \\
\hline III. II. & $\begin{array}{l}10^{\circ} \\
10 \\
15 \\
10\end{array}$ & $\begin{array}{l}15^{\circ} \\
15 \\
15 \\
15\end{array}$ & $\begin{array}{l}-5^{\circ} \\
-5 \\
0 \\
-5\end{array}$ & $\begin{array}{l}30^{\circ} \\
15 \\
20 \\
\cdots\end{array}$ & $\begin{array}{l}31^{\circ} \\
31 \\
31\end{array}$ & \begin{tabular}{c||}
$1^{\circ}$ \\
16 \\
11 \\
-
\end{tabular} & $\begin{array}{l}40^{\circ} \\
35 \\
35 \\
-\end{array}$ & $\begin{array}{l}46^{\circ} \\
46 \\
46\end{array}$ & $\begin{array}{l}-6^{\circ} \\
-11 \\
-11 \\
-\end{array}$ & \begin{tabular}{c}
$45^{\circ}$ \\
\hdashline \\
\hdashline \\
-
\end{tabular} & $58^{\circ}$ & $\begin{array}{l}-13^{\circ} \\
-\ldots . \\
-\cdots\end{array}$ \\
\hline
\end{tabular}

The observed values in the above table were taken from the composite drawing (Plate XX., Fig. 3), found in United States Naval Observatory publication on the "Solar Eclipse of July 29, 1878." The photographs taken at different stations are in accord with each other. The numerous drawings are, às usual, very discordant among themselves, but on the whole indicate a comparatively large value of $\beta$. The northern wings have 
multiple boundaries on the sides towards the nearer pole. Both coronal poles are east of the Sun's poles; but the line joining the poles of the corona, as found by means of the boundaries of the wings adjacent to the poles, is inclined towards the east, as required for a typical form. The polar rays, alone considered, would indicate that the coronal axis was nearly parallel to the Sun's axis. Professor Harkness calls attention to the fact that the orientation may be several degrees in error, as only the sides of the photographic plates could be used as guides.

The excellent drawing by Trouvenot is in remarkable agreement with the photographs. Naked-eye drawings by Professor Newcomb and Professor Langley, the latter being stationed on the top of Pike's Peak, show the greatest equatorial extent of the corona that has been observed up to the present time. (Compare the drawing made from the photographs with Figure 5, Plate VI.) (Minimum of spots, 1879.0.)

\section{0, January 11.}

The sketches made during this eclipse show how untrustworthy the data derived from mere drawings can be. In Professor Davidson's Report (see United States Coast and Geodetic Survey Report for 1882), the corona is outlined as in Figure 2 or 3, Plate VI., while in the sketches of Lieutenant Christopher only a narrow, nearly concentric band of light is shown surrounding the dark limb of the Moon. There are no photographs of this eclipse, so far as known.

\section{2, May 17.}

The drawing, made from photographs, given in Captain АвNEY's and Dr. Schuster's report on the solar eclipse, is exceedingly interesting as representing an arrangement of streamers. similar to the conditions found in the model; that is, no polar rays are formed. The general appearance of the nearly circular outline of the corona would seem to indicate that the solar activity was considerable in the higher limits of the spot-zones; or, what is more probable, that those in the middle latitudes were retarded, and that the incoming streamers were more numerous at the equator (see Postscript), and that in both zones only the more conspicuous streamers of the farther hemisphere are seen in projection through the dense masses of 
rays, most of which are apparently wholly formed in the nearer hemisphere. There is strong evidence tending to show that there were numerous collisions, as in many places the corona is brightest at some distance from the Moon's outline. I can almost exactly reproduce this coronal form with a model. The inner portions of Figure 2, Plate VI., are quite suggestive. (Minimum of spots, 1879; maximum, 1883.)

\section{3, May 6.}

The drawings made by Professor TACCHIn and Dr. Dixon give evidence of a considerable inclination of the Sun's axis. The Lick Observatory does not possess any photographs.

Note-October, 1890.- Since the above was written the 1889 volume of the "Philosophical Transactions" has been received. Like the previous eclipse (1882) there is much confusion in the coronal detail. If the orientation of the enlarged drawing made from photographs secured by the English party is correct as published in this volume, then we have here a case of a nearly circular corona, in which the less inclined streamers projected near the north pole of the Sun had a decidedly greater density than the more inclined ones; since the apparent north coronal pole is at the enormous distance of nearly $20^{\circ}$ to the east of the Sun's pole!! At the south pole the structure is more nearly radial, so that the position of the south coronal pole can not be located with great accuracy.

Note No. 2-March, 1891.-Since the above has been in type I have, very fortunately, found a photographic print of this eclipse (see Annuaire, Bureau des Longitudes, 1884), made by M. JAnssen from a negative which he exposed during the whole time of totality. Although the orientation can only be made by means of the edges of the card on which the print is mounted (the north pole is toward the lower left-hand corner of the card), still all doubt as to the proper orientation of the above mentioned drawings seems to be removed. Dr. Dixon observed with a diagonal eye-piece; the top of his drawing should therefore be marked S. instead of $\mathrm{N}$.

In Mr. Wesley's drawing (Phil. Trans.) the top of the page is $\mathrm{N}$. The axis is correctly orientated.

The remarkable difference which can exist between the drawing of a photograph taken with one instrument and a photographic print from a negative taken with another instrument is 
well illustrated in this case. In JANssen's print not only are the symmetrical wings and fish tails shown, but even the trumpetshaped outlines, on the east side at least, are unmistakable. The extended luminosity near the north pole would seem to indicate that the streamers were not uniformly distributed in longitude, so that the ratio of $D$ to $D^{\prime}$ is no longer that which holds good for a typical corona. Now, comparing Mr. WesLeY's drawing with the print, an apparent tendency to form two north and, less conspicuously, two south coronal poles adjacent to the boundaries of the wings is at once recognized. The reversal of the inclinations near the north pole is very marked and is due to an irregular variation in the density of the streamers, as can be readily shown with the aid of the models (see, for instance, Plate VII., Fig. $a$ ). The tendency to form multiple poles is also partially shown in some of the figures of Plate VI.

The axis, which is symmetrically situated with reference to these poles, in Mr. WesLEY's drawing, is inclined towards the west, in agreement with the theory. Compare the print with Figures 3, 4, and 6 of Plate VI., disregarding the unsymmetrical luminosity above the north pole. (Maximum of spots, 1883.)

\section{6, August 29.}

The photographs taken by Pickering on the Island of Grenada show enough of the finer detail in certain places near the poles to allow of a rough comparison between theory and observation. The inclinations (to the nearest, $5^{\circ}$ ) of the polar rays to normals through their bases are given in the table below for each $10^{\circ}$ of polar distance applicable. The measures are made from the drawing given in the frontispiece of Harvard College Observatory Annals, Vol. XVIII., No. V.

\begin{tabular}{|c|c|c|c|c|c|c|c|c|c|c|c|c|}
\hline \multirow{3}{*}{ QUADRANT. } & \multicolumn{12}{|c|}{ Distance from Nearer Pole. } \\
\hline & \multicolumn{3}{|c|}{$10^{\circ}$} & \multicolumn{3}{|c|}{$20^{\circ}$} & \multicolumn{3}{|c|}{$30^{\circ}$} & \multicolumn{3}{|c|}{$40^{\circ}$} \\
\hline & $o$ & $c$ & $o-c$ & $o$ & $c$ & $o-c$ & $o$ & $c$ & $o--c$ & $o$ & $c$ & $o-c$ \\
\hline $\begin{array}{l}\text { I. } \\
\text { III. } \\
\text { IIV. }\end{array}$ & $\begin{array}{l}15^{\circ} \\
15 \\
10 \\
?\end{array}$ & $\begin{array}{l}15^{\circ} \\
15 \\
15 \\
-\end{array}$ & $\begin{array}{l}0^{\circ} \\
0 \\
-5 \\
\ldots\end{array}$ & $\begin{array}{l}25^{\circ} \\
20 \\
\cdots\end{array}$ & $\begin{array}{l}31^{\circ} \\
31^{\circ}\end{array}$ & \begin{tabular}{c}
$-6^{\circ}$ \\
-11 \\
\hdashline- \\
\hdashline-
\end{tabular} & $\mid---$ & $46^{\circ}$ & $+4^{\circ}$ & $\cdots$ & . . & \\
\hline
\end{tabular}


In the second quadrant, at $30^{\circ}$ polar distance, the rays are not distinct, being nearly lost in the general illumination; the measure is therefore unreliable. Just at the south pole there is a cone with the sides inclined $10^{\circ}$ to the normal, apparently made by the overlapping of rays. On the west side of the north pole the distribution of the streamers is apparently too irregular to produce even a trace of the symmetrical inclined rays; this supposition is further strengthened by the fact that at $25^{\circ}$ polar distance there is a great wing extending to a distance nearly equal to the equatorial extent of the corona. As required by the theory when $\beta$ is positive, the axis of the inner corona is inclined towards the east. (Maximum of spots, 1883; minimum, 1888 or 1889 .)

Note-October, 1890.-Since the above was written the volume of the Phil. Trans., which contains a drawing of a photograph of this corona (discussed by Messrs. Darwin, Schuster, and Maunder), has appeared. The orientation differs something less than $4^{\circ}$ from the Prckering photograph, so that for this copy of the corona the coronal axis is slightly inclined westward. The following measures can be compared with those in the preceding table:

\begin{tabular}{|c|c|c|c|c|c|c|c|c|c|c|c|c|}
\hline \multirow{3}{*}{ QUADRANT. } & \multicolumn{12}{|c|}{ Distance from Nearfer Pole. } \\
\hline & \multicolumn{3}{|c|}{$10^{\circ}$} & \multicolumn{3}{|c|}{$20^{\circ}$} & \multicolumn{3}{|c|}{$30^{\circ}$} & \multicolumn{3}{|c|}{$40^{\circ}$} \\
\hline & $o$ & $c$ & $o-c$ & $o$ & $c$ & $o-c$ & $o$ & $c$ & $o-c$ & $o$ & $c$ & $o-c$ \\
\hline $\begin{array}{l}\text { I. } \\
\text { IIIII } \\
\text { IIV. }\end{array}$ & $\begin{array}{l}20^{\circ} \\
20 \\
10 \\
0\end{array}$ & $\begin{array}{l}15^{\circ} \\
15 \\
15 \\
15\end{array}$ & $\begin{array}{r}+5^{\circ} \\
+5 \\
-5 \\
-15\end{array}$ & $\begin{array}{l}40^{\circ} \\
30 \\
20 \\
20\end{array}$ & $\begin{array}{l}31^{\circ} \\
31 \\
31 \\
31\end{array}$ & $\begin{array}{l}+9^{\circ} \\
-1 \\
-11 \\
-11\end{array}$ & $50^{\circ}$ & $46^{\circ}$ & $\begin{array}{l}+4^{\circ} \\
-\end{array}$ & & 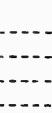 & 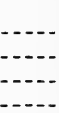 \\
\hline
\end{tabular}

If the orientation is changed so as to agree with Pickering's diagram, the above residuals will be reduced in magnitude somewhat.

1887, August 18-19.

The best photographs taken at Jourgewetz by Dr. BELOPOLSKY are similar to those of the previous year. A certain confusion is apparent, so that no great amount of detail is shown at the poles. The axis of the inner corona apparently had a 
decided inclination toward the east, as required by the theory when $\beta$ is positive. Dr. Glasenapp's results give the same evidence. (Minimum of Sun spots, probably 1888 or 1889 .)

\section{9, January 1.}

It is no exaggeration to say that the photographs taken during this eclipse are better than any which had previously been taken. On the magnificent negatives secured by BARNARD, Burckhalter, Charroppin, Harvard College Observatory party, and others, the coronal structures are shown in a way that leaves but little to be desired.

In the following table the comparison between theory and observation will prove interesting:

\begin{tabular}{|c|c|c|c|c|c|c|c|c|c|c|c|c|}
\hline \multirow{3}{*}{ QUADRANT. } & \multicolumn{12}{|c|}{ Distance from Nearer Pole. } \\
\hline & \multicolumn{3}{|c|}{$10^{\circ}$} & \multicolumn{3}{|c|}{$20^{\circ}$} & \multicolumn{3}{|c|}{$30^{\circ}$} & \multicolumn{3}{|c|}{$40^{\circ}$} \\
\hline & $o$ & $c$ & $o-c$ & $o$ & $c$ & $o-c$ & $o$ & $c$ & $o-c$ & $o$ & $c$ & $o-c$ \\
\hline \multirow{3}{*}{$\begin{array}{l}\text { I. } \\
\text { III. } \\
\text { III. } \\
\text { IV... }\end{array}$} & \multirow{3}{*}{$\begin{array}{l}20^{\circ} \\
20 \\
10 \\
10\end{array}$} & \multirow{3}{*}{$\begin{array}{l}15^{\circ} \\
15 \\
15 \\
15\end{array}$} & \multirow{3}{*}{$\begin{array}{l}+5^{\circ} \\
+5 \\
-5 \\
-5\end{array}$} & $30^{\circ}$ & $31^{\circ}$ & $-1^{\circ}$ & $35^{\circ}$ & $46^{\circ}$ & $--11^{\circ}$ & $50^{\circ}$ & $58^{\circ}$ & $-8^{\circ}$ \\
\hline & & & & 25 & 31 & -6 & $-\cdots$ & $\cdots$ & 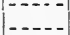 & & & - n \\
\hline & & & & 25 & 31 & -6 & 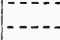 & & $-\ldots$ & - n & & 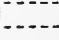 \\
\hline
\end{tabular}

I have only used those polar distances at which the individual rays could still be recognized. The corona was nearly of the typical form. The great equatorial extension with the trumpet-shaped outlines has just the form which streamers of indefinite length from low latitudes will produce in projection. With the increase of distance the illumination gradually fades out, but towards the axes of greatest density in projection the increasing number of overlapping streamers causes a gradual increase in the illumination, resulting in the trumpet-shaped outlines which form so prominent a feature of this eclipse. For all coronas, in which the fish tails are prominent, this particular feature should hereafter be shown in cases where the observers are favored with exceptionally good atmospheric conditions. This was the case for the present eclipse; the clear California sky revealed outlines not before seen on photographs taken during sun-spot minima. 
Not only is this phenomenon a necessary consequence of the theory, but all doubt as to its nature would seem to be removed by the appearance of the photographs themselves; numerous streamers, of such a length and form as to preclude the idea that they coincide with magnetic lines of force, are seen radiating from parts of the Sun's limb, which indicate that the origins are in the low latitudes. Here, again, the model need only be compared with the photographs, to test the agreement of the theory with observation. The boundaries of the wings in the southern hemisphere are multiple on the sides turned toward the nearer pole. The axis of the inner corona, as required by the typical form, is inclined toward the west, the Earth being below the Sun's equator. (Minimum of spots, 1888 or 1889.)

\section{9, December 21, 22.}

The photographs taken at Cayenne again show the structure of the corona with such minuteness of detail that a direct comparison between the observed and theoretical inclinations of the polar rays can also be used to test the theory.

As the position of the meridian is not indicated on the prints, the photographs can be accurately orientated by means of the following datum. The center of the heaviest north polar ray (the most westerly one of the more conspicuous and nearly normal rays) has a position angle of $4^{\circ} .8$; the pole of the Sun is therefore $2^{\circ}$ east of the heavy north polar ray.

\begin{tabular}{|c|c|c|c|c|c|c|c|c|c|c|c|c|}
\hline \multirow{3}{*}{ QUADRANT. } & \multicolumn{12}{|c|}{ Distance From Nearer Pole. } \\
\hline & \multicolumn{3}{|c|}{$10^{\circ}$} & \multicolumn{3}{|c|}{$20^{\circ}$} & \multicolumn{3}{|c|}{$30^{\circ}$} & \multicolumn{3}{|c|}{$40^{\circ}$} \\
\hline & $o$ & $c$ & $o-c$ & $o$ & & $o-c$ & $o$ & $c$ & $o-c$ & $o$ & $c$ & $o-c$ \\
\hline $\begin{array}{l}\text { III. } \\
\text { III. } \\
\text { IV. }\end{array}$ & $\begin{array}{l}10^{\circ} \\
10 \\
20 \\
20\end{array}$ & $\begin{array}{l}15^{\circ} \\
15 \\
15 \\
15\end{array}$ & $\begin{array}{l}-5^{\circ} \\
-5 \\
+5 \\
+5\end{array}$ & $\begin{array}{l}20^{\circ} \\
25 \\
35\end{array}$ & $\begin{array}{l}31^{\circ} \\
31 \\
31\end{array}$ & $\mid$\begin{tabular}{l}
$-11^{\circ}$ \\
\hdashline- \\
+4
\end{tabular} & $\begin{array}{l}40^{\circ} \\
-45 \\
45\end{array}$ & $\begin{array}{l}46^{\circ} \\
46 \\
46\end{array}$ & $\begin{array}{l}-6^{\circ} \\
-1 \\
-1\end{array}$ & & & \\
\hline
\end{tabular}

Although the sky was apparently free from clouds during the total phase of the eclipse, the air was so charged with moisture that the atmospheric glare must have been very prominent. Yet, notwithstanding these conditions, the equatorial extent of the corona, as shown on the photographs, is more than a degree; 
the wings are very conspicuous, and the polar rays are shown with all desired distinctness. The trumpet-shaped outlines, so prominent a feature in the previous eclipse, are lost in the sky illumination, although faint traces of this phenomenon can be distinguished.

In the southern zone there was evidently a vigorous outburst near longitude $\tau-\tau_{0}=0^{\circ}$. The protuberances (shown on the negatives) projected at this part of the Moon's outline give evidence to the same effect. The boundaries of the wings in the southern hemisphere are multiple on the sides turned towards the nearer pole.

As required by the theory, the axis of the inner corona is inclined slightly towards the west, the Earth being below the Sun's equator. (Minimum of spots, 1888 or 1889.)

\section{Probable Form of the Solar Coronas of 1892 and 1893.}

The next total eclipse of the Sun takes place in 1892, April 26. If observed, the following prediction as to its form should, in general, be verified, under favorable atmospheric conditions: Polar extent large. Inner coronal axis (if polar rays or wings are shown) inclined westward. Rifts numerous. Indications of collisions. General form similar to Figure 7, Plate VI. The same description will apply to the eclipse of April 15-16, 1893.

It will be noticed that I have used the same constants for finding the value of $k$ for a given $p$ in all cases (see page 75). A closer agreement between theory and observation could doubtless have been obtained by varying the values of $c$ and $a$ (for different values of $\beta$ ) and taking into account the shifting of the coronal pole; but as it appeared that the measured inclinations were liable to errors, which as a rule much exceeded those due to neglecting the terms which involved or caused the shifting of the coronal poles, I deemed it best to refer all measures to the Sun's true poles. The magnitude of the residuals obtained can then be used to detect any abnormal discrepancies which may result from some error in the orientation of the photograph.

The object of the present investigation was not to force an exact agreement by the arbitrary variation of certain quantities, but to secure confirmatory or non-confirmatory evidence, 
by comparing a purely theoretical investigation of a general character with the results obtained from actual observation.

It is hardly necessary to state that nothing in this theory requires that all the streamers shall be confined between any fixed circles of latitude, nor that the apparent density and magnitude of the streamers may not be subject to periodic variations, due to physical causes which relate to the molecular nature of the Sun's internal constitution, with which this investigation has nothing to do. (See Postscript.)

There seems to be no feature of the coronal structure which cannot be accounted for in a satisfactory manner by this new theory of the solar corona.

In conclusion, it gives me pleasure to thank Messrs. BurNHAM and BARNARD for their aid in the work of the photographic reproductions, and Mr. KEELER for various information relating to spectroscopic solar physics.

I am much indebted to Professor Holden, who, during the preparation of this memoir, has ever been ready to aid me in various ways.

Mт. Hamilton, June, 1890.

J. M. Schaeberle.

\section{POSTSCRIPT.}

I have spent some time in an extended examination of the magnificent spectroscopic observations made at Rome, with a view to determine, if possible, whether the solar prominences observed in high latitudes could not be reconciled to the hypothesis that they were but the projections of streams, which, issuing from a low latitude, appeared in projection to come from the limb of the Sun. In many instances this seems to be the case, but the complications, especially in the low latitudes, resulting from the perspective overlapping of these prominences in largely different longitudes, is so great that much uncertainty exists as to the motion of any given prominence. The uncertainty could be settled at once by following a conspicuous polar prominence for several hours; and carefully recording its position-angle from time to time. If the base of such a prominence is in a low latitude, the position-angle 
should change at the rate of about $2^{\circ}$ an hour. I have not had an opportunity to make an observation of this kind since the theoretical investigation was completed.

The prominences at the poles are so rare, and the observations usually so short, that no definite conclusions can be drawn from the data at hand. Again, prominences at the poles should, on this hypothesis, always have large component velocities either towards or away from the Earth.

During the total eclipse of August 29, 1886, Professor SchusTER observed the spectrum of a great prominence near the pole of the Sun. His observations indicated a motion of two hundred and forty-seven miles per second towards the observer. Assuming the origin of the prominence to be in the spot-zone, this velocity indicates roughly an initial parabolic velocity.

As bearing upon this point, the following extracts from "Chemistry of the Sun," by that eminent solar physicist, J. Norman Lockyer, will be found very suggestive. The italics are my own:

Such prominences have been seen to mount upwards at the rate of 250 miles a second; that is, nearly 1,000,000 miles an hour. * * * There are indications that these prominences, instead of rising vertically, as we may imagine them to do, are at times shot out sideways-almost tangentially. In that case, of course, the spectroscope enables us to determine the velocity. One hundred miles a second, either towards or from the eye, is by no means an uncommon velocity. * * * The height of some of these prominences is very great. Professor Young records one seen in 1878 as being nearly 400,000 miles high; that is, $13 \frac{1}{2}$ minutes of arc, the solar radius being 16 minutes.

The actual velocity of motion will, in general, always be greater than the observed, since the spectroscope only gives that component which is parallel to the Earth's radius-vector, while the direct visual observation only gives the component at right angles to the same line.

The small inclined saw-tooth protuberances, as well as many of the grotesque larger ones, are probably formed in much the same way that the polar rays are produced; the sudden changes of inclination being due to variations in the density of the streams. It would seem to require a continuous and more or less uniform series of eruptions in certain zones on the solar surface to satisfactorily account for these ever-present smaller protuberances, and the theory certainly demands such a distribution of the streamers. Let us now consider 
Some Physical Phenomena Involved in the Mechanical, Theory of the Corona.

While I invite the severest criticism, from astronomers, of the theory given in the preceding pages, $I$ ask their indulgence with reference to certain deductions which I have made, and now, with great deference, submit to their attention. Assuming my premises (The Mechanical Theory) to be true, I trust that in deducing some of the resulting phenomena my arguments will at least be found to have a logical sequence.

All the results obtained in the foregoing investigation have been deduced on the hypothesis that the force of ejection is such as to give a parabolic velocity to the streams; but so far as the form near the Sun is concerned, an inspection of Table I. at once shows that practically the same results would be obtained on the hypothesis that the force is only just sufficient to give an elliptical velocity corresponding to a period of only a few hours.

The moment the returning streams are taken into consideration the effect is such as to cause a periodic variation in the detail of the corona, and simultaneously to cause a periodic variation in the surface features of the Sun, as I shall now proceed to show.

To whatever cause the eruptions in certain zones on the Sun may be due, we are evidently justified in assuming that, in the long run, the forces there at work have, at a given period of the Sun's age, a mean value $(F)$. One measure of this force $(F)$ will be the maximum distance $(d)$ from the Sun's center $(=2 a$ nearly), to which a given particle (mass $=m)$ is projected in the time $\frac{1}{2} t$ (nearly), so that the periodic time will be $t$. As has already been shown, the heliocentric latitude of an ejected particle during its whole motion will remain nearly the same, so that the latitudes of different parts of the same stream will be nearly the same as the latitude of the point of ejection. Now, if each stream of particles in a given zone is ejected by an instantaneous force $(F)$, the more advanced portions of the streams will be unimpeded during the first half of their path; on returning, however, the chance of collision with the same or other outgoing streams varies inversely as the square of the distance from the Sun.

Near the Sun, therefore, collisions must occur which tend to 
retard or stop the outgoing streams, resulting in a temporary increase in the heat of the combined colliding masses (causing a consequent increase in the brightness of the corona at such places, and at the same time rendering the coronal detail more confused). This heat will tend to be largely dissipated before such masses fall back into the Sun, which they will then reach with comparatively.small velocity and low temperature. Unretarded returning streams on striking the Sun will tend to greatly raise the temperature at the points of impact; perturbed returning streams could, of course, strike all parts of the Sun's surface. but the general tendency of these perturbations will be to diminish the latitudes of the returning streams. Unperturbed returning streams will always fall within the limits of the Sun-spot zones.

So long as the incoming streams are very numerous, the outgoing ones will, in a great measure, be stopped, so that, after the interval $t$, there will be comparatively few returning streams; a direct consequence of this state of things is to allow free passage for the outgoing streams, which, since there are now but few collisions, results in (1) an apparent diminution in the brightness of the corona, (2) more regular and sharply defined detail, and (3), in general, a more uniformly illuminated solar surface (i.e., fewer solar spots).

The periodic character of this phenomenon can be well illustrated by means of a vertical jet of water. When the water is first turned on it almost instantly shoots up to its maximum height; the returning drops then gradually check the velocity of the stream near the origin, so that after a moment the whole mass seems to be piled up just a little above the orifice; before the last portions of the still falling stream reach the retarded stream the jet begins to resume its former activity, and then again rises to a considerable height. These oscillations are repeated at nearly uniform intervals, the period of one complete phase being roughly equal to twice the time required for a given drop to describe the whole path.

If the ejective force is such as to make $t$ about five years, a complete cycle of changes will take place in the time $2 t$, and after the same manner as is observed in the Sun-spot cycle. It is rather remarkable that the aphelion distance of the streams corresponding to this value of $t$ is nearly the same as Jupiter's 
distance from the Sun; so that the perturbations produced by this planet may have more to do with the regularity of the period than the assumed constant force of ejection. The initial velocity required to just carry a particle from the Sun to Jupiter: is but little less than a parabolic velocity. For an initial parabolic velocity Saturn, alone considered, would, on the same hypothesis, cause a complete cycle of less marked changes in twenty years, Uranus in sixty years, and Neptune in one hundred and twenty years. The comparatively insignificant planets inside of the orbit of Jupiter would cause minor variations, corresponding to cycles, which, even for Mars, would be of less than two years' duration.

A maximum of Sun spots will therefore correspond to the times when the returning streams are most numerous; the corona at these same times will be brightest and most confused near the Sun, and on account of the retardations, the equatorial extent will, as a rule, be least. But at the equator. there will be more returning than outgoing streams (on account of the planetary perturbations); consequently, the illumination in this plane will be greater at this time than it is when only outgoing streams are present.

A minimum of Sun spots will correspond to the times when the incoming streams have been exhausted. Consequently, the unimpeded outgoing streams will have their normal velocity and extent, the corona will show great equatorial extension, and, as a rule, be more sharply defined and have more prominent fish-tail outlines, especially when the Earth is near the Sun's equator.

In the equation:

$$
V=\frac{F}{M}
$$

$V$ increases as $M$ diminishes. As it is probable that at the instant of ejection the masses are of a gaseous character, the velocity may be very great without necessarily requiring an improbable ejective force. It may be well to call attention to the fact that the initial velocity required to send a particle to a distance of only one solar diameter from the Sun's surface is already greater than four fifths of the velocity required to send the same particle to an infinite distance. For lines of motion 
which are not normal to the Sun's surface, much greater initial velocities will be required to carry the particles to the same distance.

On this hypothesis the duration of the Sun-spot period therefore indicates that the forces of ejection are such as to give the streams a mean velocity but. little less than that in a parabolic orbit. Streamers having inclined initial directions of motion will, as a rule, either be destroyed by others, or be so scattered that no marked effects will be produced by them. (The zone of maximum Sun spots is the same as the zone above which the maximum number of collisions takes place.) As the Earth's maximum distance from the plane of the Sun's equator is less than $8^{\circ}$, and as the zone of maximum activity on the Sun has twice this latitude, the chance of the Earth passing through an outgoing stream is less than it is for an incoming (perturbed) stream. Such encounters must, however, take place.

\section{The Zodiacal Light and the Gegenschein.}

According to the Mechanical Theory, the ejected streams of matter, although they are of double curvature, will always be directed nearly towards the Sun (except at aphelion). Let us now consider some phenomena which can be produced experimentally. The diffused light caused by a series of nearly parallel luminous lines (of indefinite extent) very distant from the observer will always be most conspicuous in those directions in which the lines are projected as mere points. If the observer is placed within the space through which these lines pass, there will be two points of equal maxima $180^{\circ}$ apart; but if the actual intrinsic brilliancy of the lines increases from one extremity of the set to the other, then, of course, the two maxima will differ enormously in brilliancy. If the depth of these lines in cross-section is the same on all sides of the observer, the illumination will be symmetrically arranged in concentric zones, which gradually decrease in brightness as the angular distance from their common pole (which corresponds to the brighter maximum) increases, until the angular distance is reached at which the apparent increase in brilliancy due to projection more than compensates for the decrease in brilliancy due to distance from the origin. If one diameter of the crosssection at the observer is greater than any other, the excess in depth produces an apparent increase of illumination in a plane 
which contains this diameter and the vanishing points of the lines, the law of variation of brightness in this plane being the same as for a circular cross-section. If the observer is near, but not within, the space traversed by the lines of indefinite extent, no conspicuous variations will be apparent, although one hemisphere will actually be brighter than the other. If in each of the above cases the luminous lines be assumed to radiate from a common center, which is at a great distance from the point of observation, the distribution of those lines which pass nearest to the observer will, on account of their small distance, almost wholly determine the form of the resulting phenomenon.

Let us now consider the phenomenon caused by the outgoing and incoming streamers from the Sun. In December and June the Earth is in the plane of the Sun's equator and at its maximum distance from the nearest zone of streamers, and it is symmetrically situated with reference to both zones. Now, since the Earth is in neither of these zones, no great contrasts of light will be produced on the side opposite to the Sun. Even if there were no streamers near the Earth, the light in the two hemispheres would be so diffused (covering the whole visible sky with the exception of an ill-defined less luminous band along the ecliptic) that it is quite probable the variations in light would not be apparent, except at the time of an eclipse of the Sun, when typical coronal forms would be seen. But the arrangement of the comparatively few streamers which pass near the Earth on all sides will, on account of their nearness, now determine the resulting form of the outlines. The density in projection will evidently be greatest in the plane of the Sun's equator, but this density will be at a minimum.

When the Earth is at its greatest distance above (September) or below (March) the plane of the Sun's equator, it will be at its least distance from the zone of maximum density of the streamers. In projection, the density will now be greatest in a plane which is practically coincident with the plane of the ecliptic; the excess of streamers in the direction of maximum density will, however, have a tendency to cause the center of illumination to be shifted slightly above the plane of the ecliptic in March and slightly below this plane in September. (The effect of the decrease in latitude of the returning streams will have a precisely similar tendency.) In general, at any time of the year, 
the illumination at any angular distance from the Sun will be greatest near a plane which contains the radius-vector of the Earth, and which is perpendicular to the orthographic projection of the Sun's axis.

The phenomenon will be least conspicuous in December and June, and most conspicuous in September and March. It will always be brightest near the Sun, and gradually diminish in intensity to within a certain distance of the point which is $180^{\circ}$ from the Sun, where theoretically there will be a slight increase in the luminosity. These conditions are in agreement with actual observation.

Now, the effect of the perturbations of the superior planets is always such that the latitude of a normally ejected returning stream will be less than that of the same outgoing stream; consequently, these streams will be more conspicuous to an observer in a smaller heliocentric latitude than the outgoing streams. If lines are drawn from the Earth tangent to the incoming stream, these lines will all have a slightly less longitude than that of the Earth, so that the center of the secondary maximum will always be west of the point which is $180^{\circ}$ from the Sun, if the returning stream is more conspicuous than the outgoing one, since lines from the Earth drawn tangent to the outgoing stream will always lie east of the same point. The greatest extent will be in longitude.

To show how this theory agrees with observation, I give below a resume of the published results of the most noted observers of the Gegenschein. I have taken the mean results for two series, one corresponding to the months when the Earth was above the Sun's equator, the other when it was below this plane:

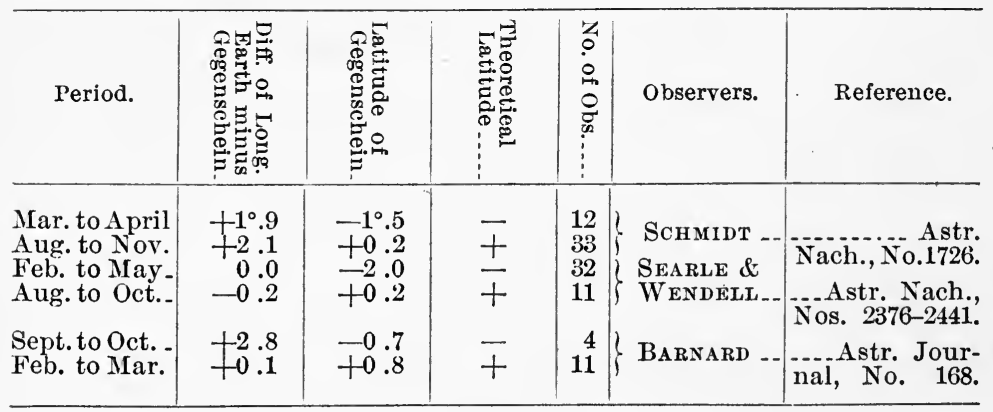


While the individual observations on any given day are often very discordant, the above mean results certainly show a remarkable agreement with the theory.

When one considers that the area covered by the Gegenschein is often as much as $20^{\circ}$ long by $10^{\circ}$ or $15^{\circ}$ wide, the difficulty of accurately locating the center of such a faint object, the caution with which apparently confirmatory evidence should be regarded, can be better understood.

In any given latitude the effect of differential atmospheric absorption of light will always have a tendency to apparently shift the center of illumination towards the zenith of the place. In northern latitudes (greater than $23^{\circ} .5$ ) the apparent displacement due to atmospheric absorption will always be greater in June than in December, and about the same in March and September.

Since there appears to be no marked parallax for the Gegenschein, and since the absence of strong solar light within the Earth's shadow appears to have no sensible effect on the central portions of this illumination, it follows that much the greater portion of the matter composing the streams must be much farther away from the Earth than the length of the Earth's shadow.

Further observations, and a much more extended comparison between theory and existing observations, are of course necessary before definite conclusions can be drawn. In a general way, however, I have, as it appears to me, conclusively shown that a phenomenon similar in form and position to the observed Zodiacal Light and Gegenschein must necessarily be produced by ejected particles whose orbits have a major axis greater than the Earth's radius-vector and whose theoretical perihelion is near the Sun's center.

\section{Forward Drift of the Solar Surface.}

- The planetary perturbations in longitude will always be such, that while the eccentricity of the orbits of some of the particles will be increased (even to the extent of causing a retrograde motion), by far the greater number, especially those having small latitudes, will become less eccentric, so that the resultant effect is to cause the returning masses to strike the Sun with a 
greater angular velocity than that of the Sun's surface, thus causing an actual forward drift of this surface, which will be greatest in the Sun's equatorial regions, in agreement with observation. (A variation in the angular distance of the Gegenschein from the Earth's radius-vector produced is also a function of the planetary perturbations in longitude.)

\section{Terrestrial Magnetism.}

We know from actual observation that the atmosphere of our planet is continually bombarded by meteoric matter, which is often volatilized before it reaches the Earth's surface. If we

= imagine the Earth to be in the center of a stream of uniformly distributed matter having a rectilinear motion in a given direction, it is at once evident that owing to the rotation of the Earth the maximum amount of meteoric matter will fall at the place which has the least linear velocity for a given inclination of the local horizon to the direction of the stream's motion, so that at the equator the amount of matter per unit of the area will be at a minimum, while a very decided maximum will be at each pole. Even for the case when the path of the stream is parallel to the Earth's equator, the area about the poles will, on account of the Earth's mass, still receive more foreign matter than a corresponding area nearer the equator, since particles will not only be deflected from the more distant portions of the stream so as to strike the areas near the poles, but the new instantaneous orbits described by these more distant particles will be such that even the region beyond the poles will be bombarded. If either pole is inclined towards the direction from which the stream is coming, the amount of matter received at that pole will be enormously greater than it will for a corresponding area at the equator. If the surface on which the matter falls is permanent, there will be a continual accumulation of such matter; if, on the other hand, the surface is a movable one, like water or a moving field of ice, the matter does not necessarily accumulate at the place, since ocean currents, sooner or later, will cause a general distribution over the globe.

Now, if we look at a map of the Earth, we.find that, beyond a certain distance, the north pole is almost completely surrounded by land; the Arctic Ocean bounds the northern coast of Europe, 
Asia, and Alaska. But from Eastern British America towards the pole the whole area is practically land-locked, so that this area will contain the greatest amount of accumulated meteoric matter. The most northerly portions of Siberia will come next in order. At the south pole practically the whole Antarctic region is still unexplored; but while the areas of maximum amount of accumulated matter cannot be accurately located, it is at once evident that the total area over which the mass of meteoric matter can accumulate will be much greater in the Northern than in the Southern Hemisphere.

Now, the larger meteoric masses which reach the Earth's surface are found, on examination, to be strongly magnetic, and the smaller masses are undoubtedly magnetic also. Hence, the arrangement of individual meteoric particles covering a given surface will be such that each one naturally assumes a fixed position with reference to the resultant lines of magnetic force. Of the two poles formed, the one in the Antarctic region will (in agreement with observation) show the greatest force, since the space into which the lines of force are condensed will be least in the Southern Hemisphere. As is also well known from actual observation, there are two areas or centers of maximum force near each magnetic pole; at least such is the case for the Northern Hemisphere, where the area of greater maximum of force is in Northern British America, while the lesser maximum is in Northern Siberia. A possible shifting of the immense ice fields at both poles, of course involves a corresponding movement of the meteoric matter imbedded in these fields, and a consequent shifting of the centers of force results. The direction of the Earth's magnetic lines of force should, according to this theory, be largely influenced by the size, outlines, and locations of the continents of the globe; and a summary inspection of a magnetic chart of the globe indicates that such is actually the case.

\section{The Aurora.}

Let us now consider the phenomena produced by the passage of the Earth through a particular one of the streams ejected from the Sun. In certain longitudes, at a given instant of time, the direction of the stream will be more nearly at right angles to the direction of the Earth's magnetic lines of force than in 
other longitudes. Now, in the case of a permanent magnet, wound by a coil of some conducting material in the form of an insulated wire, an electric discharge can be produced between the terminal points of this wire, if a magnetic body is moved rapidly across the lines of force of the magnet; the discharge will take place along the line of least resistance. . Applying this principle to the case of the Earth and the stream, then, if my reasoning is sound, it would seem to follow (1) that there will be a tendency for a discharge to take place between the particles of the stream, and that (2) such a discharge will be in the direction of the Earth's magnetic meridian; since each individual particle of the stream, on entering the Earth's magnetic field, will at once take such a position that the line joining its own poles will lie in the direction of the magnetic meridian, and as these same particles will act as conductors, the lines of least resistance will be formed in the magnetic meridians.

If we take it for granted that there is an electrostatic condition in which a positively electrified stratum is separated from one negatively electrified by an insulating stratum of air, then a much simpler explanation results. The lines of least resistance will be formed precisely as in the first hypothesis; these lines joining two differently charged strata will evidently be shortest (and the discharges therefore most brilliant) at the magnetic poles, while near the equator no discharges can take place, since any given line of least resistance will lie wholly within a given stratum. The intensity of the discharge will decrease as the area over which the discharges take place increases. When the number of discharges is very great, we have the condition of a great number of nearly parallel luminous lines more or less inclined to each other, so that in addition to the apparent motions towards the magnetic zenith, concentric arches (rays) may be formed, which will slowly vary with variations in the arrangement of the luminous discharges. (See Plate VIII., Figures 10 and 11, inverted. The parallactic effect will depend on the distance of the display from the observer, so that the arches (rays) formed may be either convex or concave in perspective.) Many of the rapid motions may be only apparent, and due to an actual shifting of the points of perspective intersection of continuous discharges, which have a slight lateral motion. Figure 1, Plate VII., illustrates the low auroral 
arch. If the gratings are slightly shifted, this luminous arch can be changed to a dark segment. Dark segments at other altitudes are illustrated in Figures 10, 11, 12, and 13.

Gravitational and atmospheric disturbances will constantly tend to break existing conductors, and to form new ones so long as the suspended particles are sufficiently numerous.

To further test this theory, the following conditions should be fulfilled:

As the streams ejected from the Sun have the greatest density in about $15^{\circ}$ heliocentric latitude, the number of auroras should be greatest in the months corresponding to the times when the heliocentric latitude $\beta$ of the Earth is greatest, and least when latitude of the Earth is zero. As, however, a returning stream is more likely to pass near the Earth than an outgoing one (on account of the tendency of the outer planets to diminish the latitudes of all the streams), the pole which is turned towards a returning stream when the Earth is in the plane of the Sun's equator will have more decided displays than the one turned towards the Sun. The Earth is in the plane of the Sun's equator in June and December, and during the latter month the north pole attains its greatest inclination away from the Sun, so that the frequency of auroras should be more decided in the Northern Hemisphere in December than in June; the effect of the longer nights will also cause an apparently greater number of displays in December. The Earth attains its greatest heliocentric latitudes in March and September, and both poles are equally exposed to the solar influences at these times, consequently the auroras should not only be most numerous at these times, but in both months the number should be about the same. I take the following data from the article on Meteorology, Vol. XVI. of the Encyclopædia Britannica:

Monthly Frequency of European Auroras.

\begin{tabular}{r|r|r|r|r|r|r|r|r|r|r|r}
\hline Jan. & Feb. & Mar. & A pril. & May. & June. & July. & Aug. & Sept. & Oet. & Nov. & Dec. \\
\hline 229 & 307 & 440 & 312 & 184 & 65 & 87 & 217 & 405 & 497 & 285 & 225 \\
\hline
\end{tabular}

If a series of observations should be manufactured to fit this theory, the above figures could hardly be improved upon. 
I have not been able to find a series of observations for the Southern Hemisphere. It at once follows that if the streams have periods of maxima and minima of magnitude and frequency, the auroras will have similar and nearly coincident periods, the returning streams, as a rule, governing the magnitude of the display; although, when the perturbations of the streams near the Sun's surface are greatest, the slightly deflected outgoing streams, from the higher latitudes, will be more apt to cross the Earth's path. If these streams are composed of small particles, the auroral phenomena will be largely confined to the higher regions of the Earth's atmosphere. (So far as the final precipitation of such particles to the Earth is concerned, the prevailing direction of the upper atmospheric currents will have much to do with the final location of such masses on the Earth's surface, and here, again, the tendency will be to carry these masses towards the poles, if our present views on the currents of the upper atmosphere are correct.)

The monthly variation in auroral frequency is thus accounted for. The variation in the absolute numbers of the auroras, taking the whole world together, will depend upon the number of the streams, and will, therefore, pass through a cycle every ten or eleven years, precisely as the Sun spots do, according to the theory, and in fact.

\section{Variable and Nebulous Stars.}

It seems to me that many of the irregular variations in the physical appearances of certain classes of stars can be satisfactorily explained by the theory of ejected streams colliding at certain intervals with the returning ones. Viewed from great distances such stars would appear to be surrounded by nebulous envelopes of varying brightness, and according to the position of the axis of rotation and the zones of maximum activity with reference to the line of sight the nebulosity would appear to be either circular or more or less elliptical in outline. The bearing of this theory on Professor Lockyer's Meteoritic Hypothesis is significant (so far as I can learn from mere notices of his work just issued), since these results have been obtained without any preconceived notions as to whether the explanation of certain phenomena would agree or disagree with any other theory. 


\section{Comets.}

The hypothesis, favored by some astronomers, that the matter now revolving about the Sun, in cometary orbits, was once ejected from the Sun, is, according to the Mechanical Theory, rendered extremely probable, and it would not be difficult to bring forward many strong arguments to support this view and to account for many apparent changes of form in cometary matter. But the length of this postscript has already far exceeded my original intentions, and I now await the result of the final verdict as to the merits of my investigations, to be given by those who are most competent to pass judgment. 



Photomount Pamphlet Binder

Gaylord Bros. Makers

Stockton, Calif. PAT. JA.N. 21, 1908

UNIVERSITY OF CALIFORNIA LIBRARY

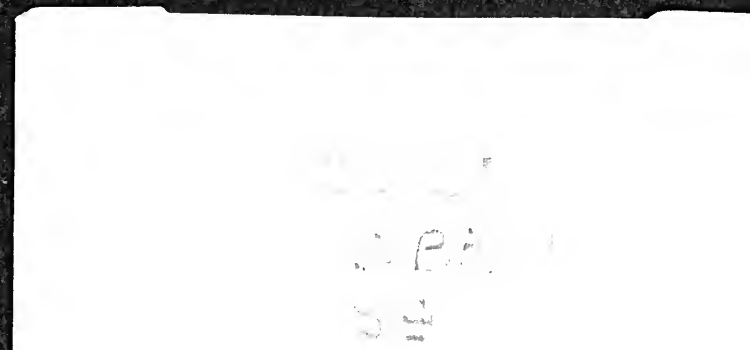




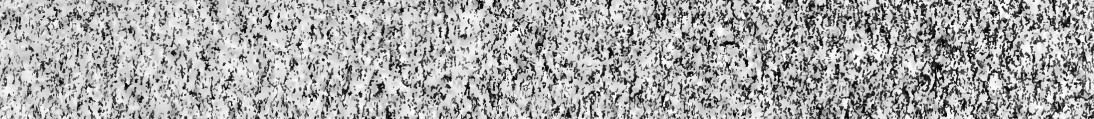

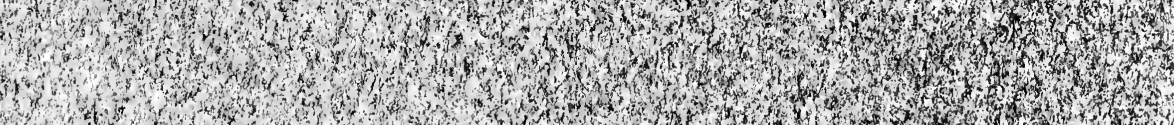
H.

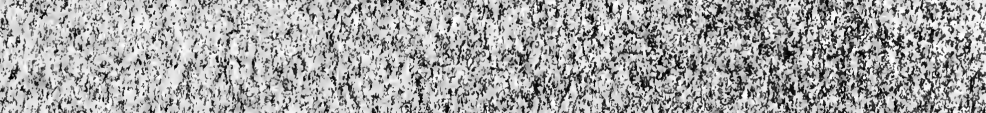

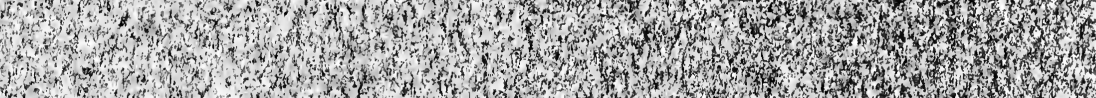

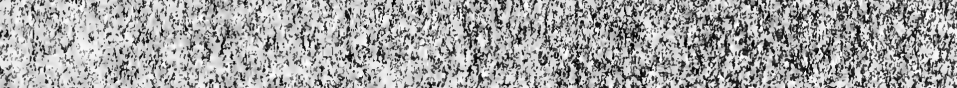

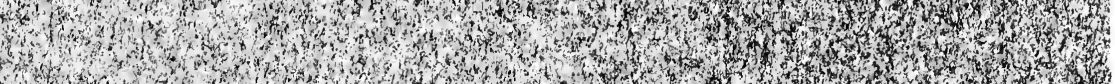

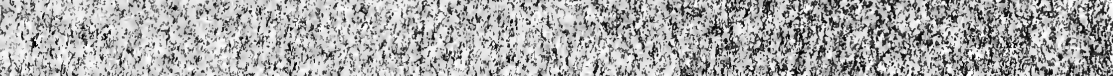

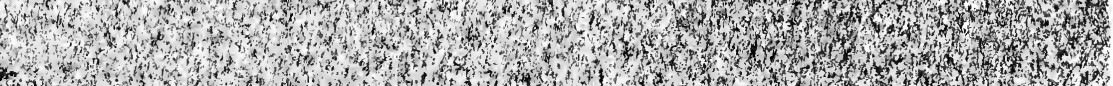

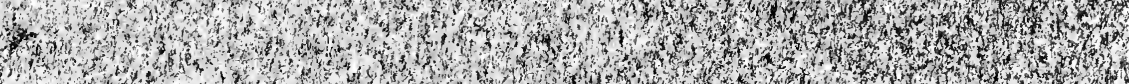
30
3 P.t.

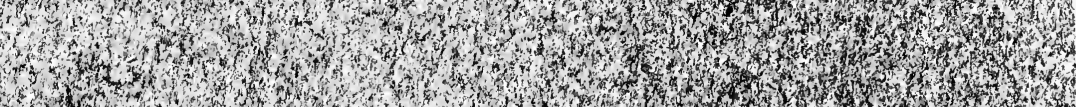

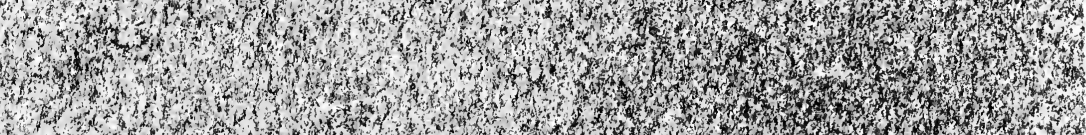

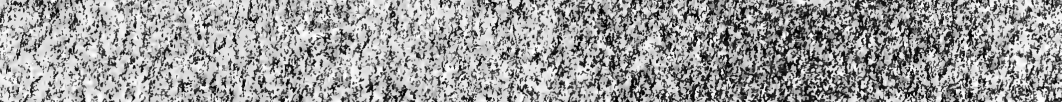

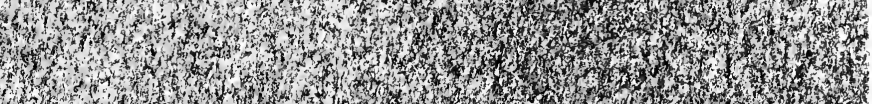

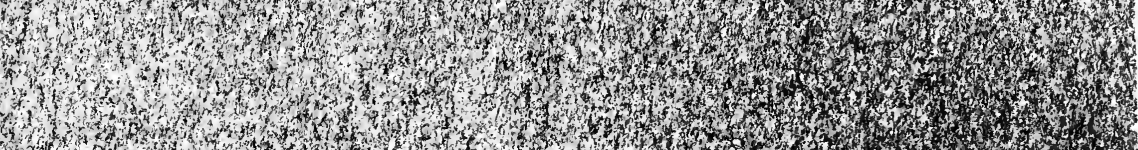

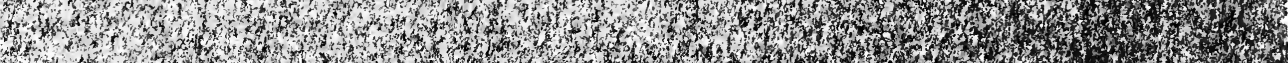

